\title{
The Evolutionary Dynamics of Direct Phenotypic Overdominance: Emergence Possible, Loss Probable
}

Tom J.M. Van Dooren (vdooren@uia.ua.ac.be)

\section{Approved by}

Ulf Dieckmann (dieckman@iiasa.ac.at)

Project Coordinator, Adaptive Dynamics Network

December 2000 


\section{IIASA STUDIES IN ADAPTIVE DYNAMICS NO. 49}

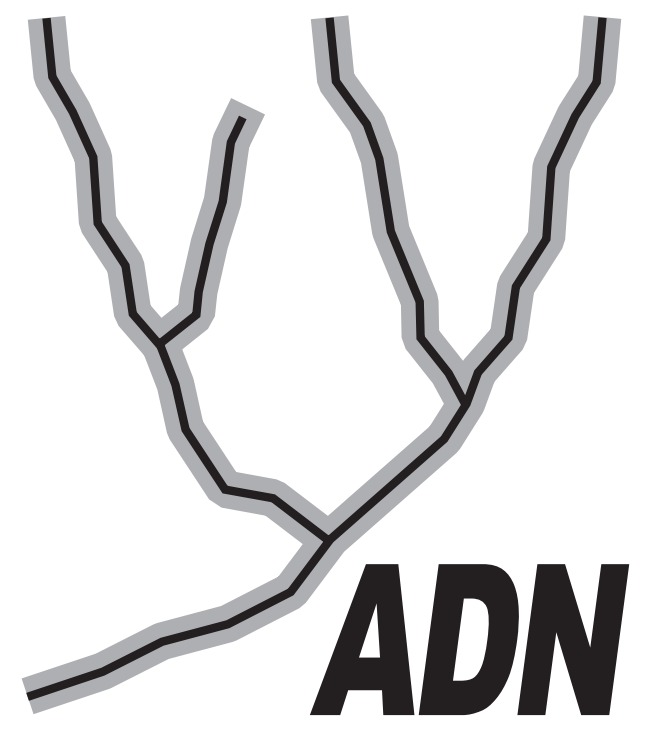

The Adaptive Dynamics Network at IIASA fosters the development of new mathematical and conceptual techniques for understanding the evolution of complex adaptive systems.

Focusing on these long-term implications of adaptive processes in systems of limited growth, the Adaptive Dynamics Network brings together scientists and institutions from around the world with IIASA acting as the central node.

Scientific progress within the network is reported in the IIASA Studies in Adaptive Dynamics series.

\section{THE ADAPTIVE DYNAMICS NETWORK}

The pivotal role of evolutionary theory in life sciences derives from its capability to provide causal explanations for phenomena that are highly improbable in the physicochemical sense. Yet, until recently, many facts in biology could not be accounted for in the light of evolution. Just as physicists for a long time ignored the presence of chaos, these phenomena were basically not perceived by biologists.

Two examples illustrate this assertion. Although Darwin's publication of "The Origin of Species" sparked off the whole evolutionary revolution, oddly enough, the population genetic framework underlying the modern synthesis holds no clues to speciation events. A second illustration is the more recently appreciated issue of jump increases in biological complexity that result from the aggregation of individuals into mutualistic wholes.

These and many more problems possess a common source: the interactions of individuals are bound to change the environments these individuals live in. By closing the feedback loop in the evolutionary explanation, a new mathematical theory of the evolution of complex adaptive systems arises. It is this general theoretical option that lies at the core of the emerging field of adaptive dynamics. In consequence a major promise of adaptive dynamics studies is to elucidate the long-term effects of the interactions between ecological and evolutionary processes.

A commitment to interfacing the theory with empirical applications is necessary both for validation and for management problems. For example, empirical evidence indicates that to control pests and diseases or to achieve sustainable harvesting of renewable resources evolutionary deliberation is already crucial on the time scale of two decades.

The Adaptive Dynamics Network has as its primary objective the development of mathematical tools for the analysis of adaptive systems inside and outside the biological realm. 


\section{IIASA STUDIES IN ADAPTIVE DYNAMICS}

No. 1 Metz JAJ, Geritz SAH, Meszéna G, Jacobs FJA, van Heerwaarden JS:

Adaptive Dynamics: A Geometrical Study of the Consequences of Nearly Faithful Reproduction.

IIASA Working Paper WP-95-099.

In: van Strien SJ, Verduyn Lunel SM (eds.): Stochastic and Spatial Structures of Dynamical Systems, Proceedings of the Royal Dutch Academy of Science (KNAW Verhandelingen), North Holland, Amsterdam, pp. 183-231 (1996).

No. 2 Dieckmann U, Law R:

The Dynamical Theory of Coevolution: A Derivation from Stochastic

Ecological Processes.

IIASA Working Paper WP-96-001.

Journal of Mathematical Biology (1996) 34, 579-612.

No. 3 Dieckmann U, Marrow P, Law R:

Evolutionary Cycling of Predator-Prey Interactions: Population Dynamics and the Red Queen.

Journal of Theoretical Biology (1995) 176, 91-102.

No. 4 Marrow P, Dieckmann U, Law R:

Evolutionary Dynamics of Predator-Prey Systems: An Ecological

Perspective.

IIASA Working Paper WP-96-002.

Journal of Mathematical Biology (1996) 34, 556-578.

No. 5 Law R, Marrow P, Dieckmann U:

On Evolution under Asymmetric Competition.

IIASA Working Paper WP-96-003.

Evolutionary Ecology (1997) 11, 485-501.

No. 6 Metz JAJ, Mylius SD, Diekmann O:

When Does Evolution Optimise? On the Relation between Types of Density

Dependence and Evolutionarily Stable Life History Parameters.

IIASA Working Paper WP-96-004.

No. 7 Ferrière R, Gatto M:

Lyapunov Exponents and the Mathematics of Invasion in Oscillatory or Chaotic Populations.

Theoretical Population Biology (1995) 48, 126-171.

No. 8 Ferrière R, Fox GA:

Chaos and Evolution.

Trends in Ecology and Evolution (1995) 10, 480-485. 
No. 9 Ferrière R, Michod RE:

The Evolution of Cooperation in Spatially Heterogeneous Populations.

IIASA Working Paper WP-96-029.

American Naturalist (1996) 147, 692-717.

No. 10 Van Dooren TJM, Metz JAJ:

Delayed Maturation in Temporally Structured Populations with Non-Equilibrium Dynamics.

IIASA Working Paper WP-96-070.

Journal of Evolutionary Biology (1998) 11, 41-62.

No. 11 Geritz SAH, Metz JAJ, Kisdi É, Meszéna G:

The Dynamics of Adaptation and Evolutionary Branching.

IIASA Working Paper WP-96-077.

Physical Review Letters (1997) 78, 2024-2027.

No. 12 Geritz SAH, Kisdi É, Meszéna G, Metz JAJ:

Evolutionarily Singular Strategies and the Adaptive Growth and Branching of the Evolutionary Tree.

IIASA Working Paper WP-96-114.

Evolutionary Ecology (1998) 12, 35-57.

No. 13 Heino M, Metz JAJ, Kaitala V:

Evolution of Mixed Maturation Strategies in Semelparous Life-Histories: the Crucial Role of Dimensionality of Feedback Environment.

IIASA Working Paper WP-96-126.

Philosophical Transactions of the Royal Society of London Series B (1997) 352, 16471655.

No. 14 Dieckmann U:

Can Adaptive Dynamics Invade?

IIASA Working Paper WP-96-152.

Trends in Ecology and Evolution (1997) 12, 128-131.

No. 15 Meszéna G, Czibula I, Geritz SAH:

Adaptive Dynamics in a Two-Patch Environment: a Simple Model for

Allopatric and Parapatric Speciation.

IIASA Interim Report IR-97-001.

Journal of Biological Systems (1997) 5, 265-284.

No. 16 Heino M, Metz JAJ, Kaitala V:

The Enigma of Frequency-Dependent Selection.

IIASA Interim Report IR-97-061.

Trends in Ecology and Evolution (1998) 13, 367-370. 
No. 17 Heino M:

Management of Evolving Fish Stocks.

IIASA Interim Report IR-97-062.

Canadian Journal of Fisheries and Aquatic Sciences (1998) 55, 1971-1982.

No. 18 Heino M:

Evolution of Mixed Reproductive Strategies in Simple Life-History Models.

IIASA Interim Report IR-97-063.

No. 19 Geritz SAH, van der Meijden E, Metz JAJ:

Evolutionary Dynamics of Seed Size and Seedling Competitive Ability.

IIASA Interim Report IR-97-071.

Theoretical Population Biology (1999) 55, 324-343.

No. 20 Galis F, Metz JAJ:

Why are there so many Cichlid Species? On the Interplay of Speciation and Adaptive Radiation.

IIASA Interim Report IR-97-072.

Trends in Ecology and Evolution (1998) 13, 1-2.

No. 21 Boerlijst MC, Nowak MA, Sigmund K:

Equal Pay for all Prisoners. / The Logic of Contrition.

IIASA Interim Report IR-97-073.

AMS Monthly (1997) 104, 303-307.

Journal of Theoretical Biology (1997) 185, 281-294.

No. 22 Law R, Dieckmann U:

Symbiosis without Mutualism and the Merger of Lineages in Evolution.

IIASA Interim Report IR-97-074.

Proceedings of the Royal Society of London Series B (1998) 265, 1245-1253.

No. 23 Klinkhamer PGL, de Jong TJ, Metz JAJ:

Sex and Size in Cosexual Plants.

IIASA Interim Report IR-97-078.

Trends in Ecology and Evolution (1997) 12, 260-265.

No. 24 Fontana W, Schuster P:

Shaping Space: The Possible and the Attainable in RNA Genotype-Phenotype Mapping.

IIASA Interim Report IR-98-004.

Journal of Theoretical Biology (1998) 194, 491-515.

No. 25 Kisdi É, Geritz SAH:

Adaptive Dynamics in Allele Space: Evolution of Genetic Polymorphism by Small Mutations in a Heterogeneous Environment.

IIASA Interim Report IR-98-038.

Evolution (1999) 53, 993-1008. 
No. 26 Fontana W, Schuster P:

Continuity in Evolution: On the Nature of Transitions.

IIASA Interim Report IR-98-039.

Science (1998) 280, 1451-1455.

No. 27 Nowak MA, Sigmund K:

Evolution of Indirect Reciprocity by Image Scoring. / The Dynamics of Indirect Reciprocity.

IIASA Interim Report IR-98-040.

Nature (1998) 393, 573-577.

Journal of Theoretical Biology (1998) 194, 561-574.

No. 28 Kisdi É:

Evolutionary Branching Under Asymmetric Competition.

IIASA Interim Report IR-98-045.

Journal of Theoretical Biology (1999) 197, 149-162.

No. 29 Berger U:

Best Response Adaptation for Role Games.

IIASA Interim Report IR-98-086.

No. 30 Van Dooren TJM:

The Evolutionary Ecology of Dominance-Recessivity

IIASA Interim Report IR-98-096.

Journal of Theoretical Biology (1999) 198, 519-532.

No. 31 Dieckmann U, O'Hara B, Weisser W:

The Evolutionary Ecology of Dispersal.

IIASA Interim Report IR-98-108.

Trends in Ecology and Evolution (1999) 14, 88-90.

No. 32 Sigmund K:

Complex Adaptive Systems and the Evolution of Reciprocation.

IIASA Interim Report IR-98-100.

Ecosystems (1998) 1, 444-448.

No. 33 Posch M, Pichler A, Sigmund K:

The Efficiency of Adapting Aspiration Levels.

IIASA Interim Report IR-98-103.

Proceedings of the Royal Society of London Series B (1999) 266, 1427-1435.

No. 34 Mathias A, Kisdi É:

Evolutionary Branching and Coexistence of Germination Strategies.

IIASA Interim Report IR-99-014. 
No. 35 Dieckmann U, Doebeli M:

On the Origin of Species by Sympatric Speciation.

IIASA Interim Report IR-99-013.

Nature (1999) 400, 354-357.

No. 36 Metz JAJ, Gyllenberg M:

How Should We Define Fitness in Structured Metapopulation Models? Including an Application to the Calculation of Evolutionarily Stable Dispersal Strategies.

IIASA Interim Report IR-99-019.

Research Report A39 (1999), University of Turku, Institute of Applied Mathematics, Turku, Finland.

No. 37 Gyllenberg M, Metz JAJ:

On Fitness in Structured Metapopulations.

IIASA Interim Report IR-99-037.

Research Report A38 (1999), University of Turku, Institute of Applied Mathematics, Turku, Finland.

No. 38 Meszéna G, Metz JAJ:

Species Diversity and Population Regulation: The Importance of Environmental Feedback Dimensionality.

IIASA Interim Report IR-99-045.

No. 39 Kisdi É, Geritz SAH:

Evolutionary Branching and Sympatric Speciation in Diploid Populations.

IIASA Interim Report IR-99-048.

No. 40 Ylikarjula J, Heino M, Dieckmann U:

Ecology and Adaptation of Stunted Growth in Fish.

IIASA Interim Report IR-99-050.

Evolutionary Ecology (1999) 13, 433-453.

No. 41 Nowak MA, Sigmund K:

Games on Grids.

IIASA Interim Report IR-99-038.

In: Dieckmann U, Law R, Metz JAJ (eds.): The Geometry of Ecological Interactions: Simplifying Spatial Complexity, Cambridge University Press, Cambridge, UK, pp. 135150 (2000).

No. 42 Ferrière R, Michod RE:

Wave Patterns in Spatial Games and the Evolution of Cooperation.

IIASA Interim Report IR-99-041.

In: Dieckmann U, Law R, Metz JAJ (eds.): The Geometry of Ecological Interactions: Simplifying Spatial Complexity, Cambridge University Press, Cambridge, UK, pp. 318332 (2000).

No. 43 Kisdi É, Jacobs FJA, Geritz SAH:

Red Queen Evolution by Cycles of Evolutionary Branching and Extinction. IIASA Interim Report IR-00-030. 
No. 44 Meszéna G, Kisdi É, Dieckmann U, Geritz SAH, Metz JAJ:

Evolutionary Optimisation Models and Matrix Games in the Unified Perspective of Adaptive Dynamics.

IIASA Interim Report IR-00-039.

No. 45 Parvinen K, Dieckmann U, Gyllenberg M, Metz JAJ:

Evolution of Dispersal in Metapopulations with Local Density Dependence and Demographic Stochasticity.

IIASA Interim Report IR-00-035.

No. 46 Doebeli M, Dieckmann, U:

Evolutionary Branching and Sympatric Speciation Caused by Different Types of Ecological Interactions.

IIASA Interim Report IR-00-040.

No. 47 Heino M, Hanski I:

Evolution of Migration Rate in a Spatially Realistic Metapopulation Model.

IIASA Interim Report IR-00-044.

No. 48 Gyllenberg M, Parvinen K, Dieckmann U:

Evolutionary Suicide and Evolution of Dispersal in Structured Metapopulations.

IIASA Interim Report IR-00-056.

No. 49 Van Dooren TJM:

The Evolutionary Dynamics of Direct Phenotypic Overdominance: Emergence Possible, Loss Probable.

IIASA Interim Report IR-00-048.

Issues of the IIASA Studies in Adaptive Dynamics series can be obtained free of charge. Please contact:

Adaptive Dynamics Network

International Institute for Applied Systems Analysis

Schlossplatz 1

A-2361 Laxenburg

Austria

Telephone +43 2236 807, Telefax +43 2236 71313, E-Mail adn@iiasa.ac.at, Internet http://www.iiasa.ac.at/Research/ADN 


\section{Contents}

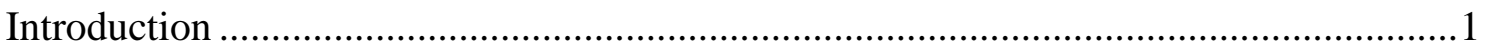

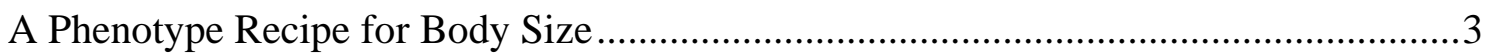

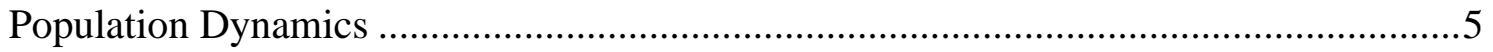

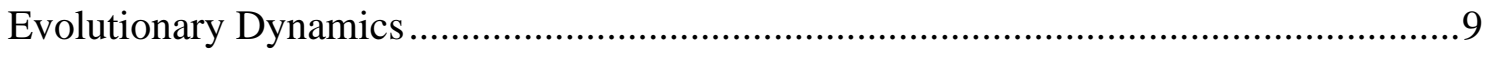

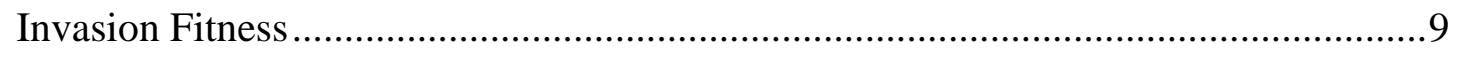

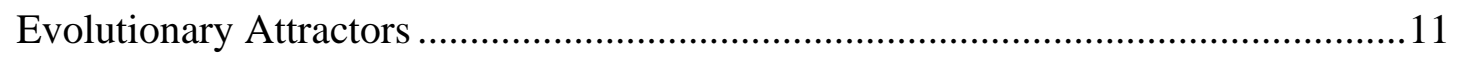

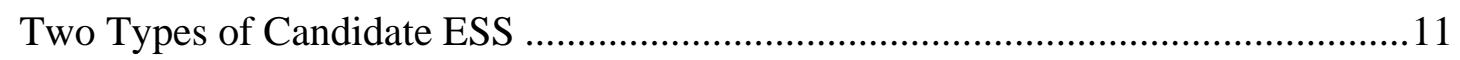

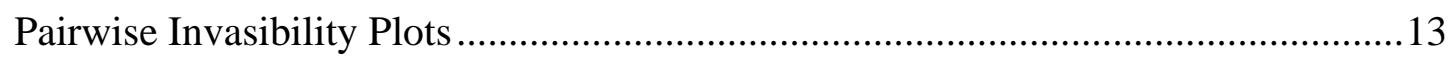

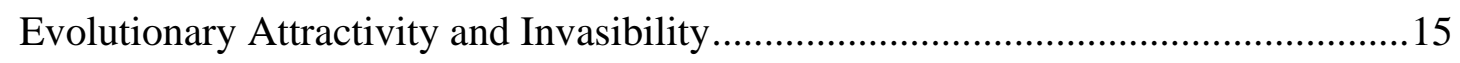

Qualitative Types of Evolutionary Dynamics and Dependence on Model Parameters

Overdominance in Single-Locus Allele Polymorphisms .......................................... 18

Mutation-Selection-Drift Balance ..................................................................... 18

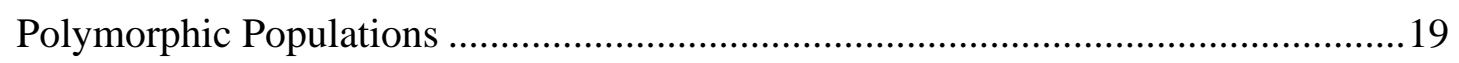

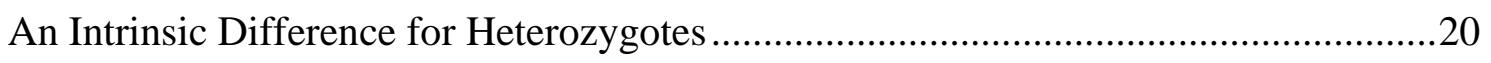

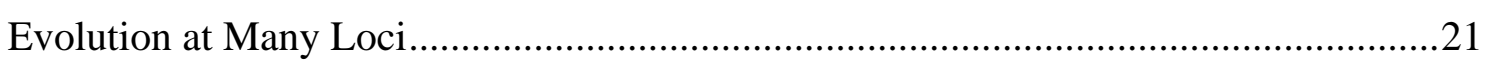

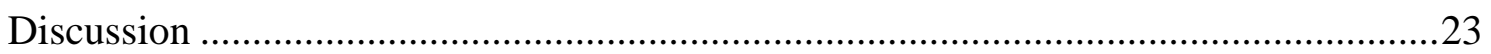

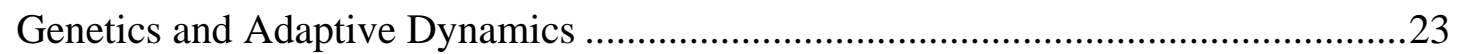

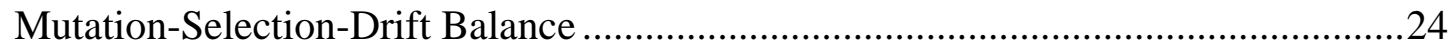

On The Likelihood of Direct Phenotypic Overdominance .......................................25

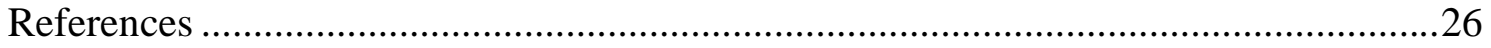

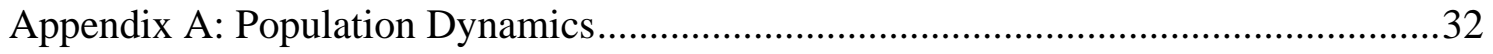

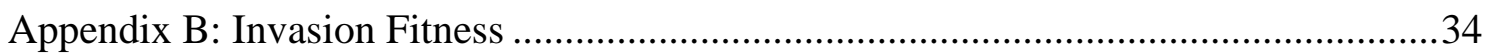




\begin{abstract}
An evolutionary dynamical system with explicit diploid genetics is used to investigate the likelihood of observing phenotypically overdominant heterozygotes vs. heterozygous phenotypes that are intermediate between the homozygotes. In this model, body size evolves in a population ecology with discrete demographic episodes and with competition limiting reproduction. A genotype-phenotype map for body size is used that can generate the two qualitative types of dominance interactions (overdominance vs. intermediate dominance). It is written as a single-locus model with one focal locus and parameters summarizing the effects of alleles at other loci. Two types of ESS (CSS) occur. The ESS is either generated (1) by the population ecology, or (2) by a local maximum of the genotype-phenotype map. Overdominant heterozygotes are expected to occur if the population evolves towards the second type of ESS, where nearly maximum body sizes occur. When other loci with partially dominant inheritance also evolve, the location of the maximum in the genotype-phenotype map repeatedly changes. It is unlikely that an evolving population will track these changes; ESS of the second type now are at best quasi-stationary states of the evolutionary dynamics. Considering the restrictions on its probability of occurrence, a pattern of phenotypic overdominance is expected to be rare.
\end{abstract}




\title{
About the Author
}

\author{
Tom J. M. Van Dooren \\ Department of Biology \\ University of Antwerp \\ Universiteitsplein 1 \\ 2610 Antwerp \\ Belgium
}

\section{Acknowledgements}

Comments by Tom Van de Casteele and an anonymous referee increased accessibility of this manuscript a lot. I thank Erik Matthysen for support. This research was carried out while I was research assistant with the Belgian FWO and with GOA-BOF-UA1999-2003. 


\section{The Evolutionary Dynamics of Direct Phenotypic Overdominance: Emergence Possible, Loss Probable}

Tom J.M.Van Dooren

\section{Introduction}

Phenotypic overdominance has not received a lot of attention in long-term evolutionary models (Maynard Smith 1981; Cressman and Hines 1984; Van Dooren in press). Extensions of phenotypic evolutionary models with diploid and sexual inheritance often assume partially dominant inheritance (e.g., Matessi and Di Pasquale 1996; Geritz et al. 1998; Kisdi and Geritz 1999), possibly because assuming small mutational effects implies approximately additive genetics in mutant heterozygotes (Barton and Turelli 1989; Van Dooren in press). Another reason for this neglect might be that direct phenotypic overdominance is not commonly observed in the field. Results from population genetic studies indicate that phenotypic overdominance is rarely caused by direct effects of a heterozygous marker locus (it is then called direct overdominance, or true overdominance) in comparison with global or associative effects of deleterious loci linked to the genetic markers (Lynch and Walsh 1998). This does not imply that direct phenotypic overdominance does not exist. Investigating the physiology of overdominance, Hall and Wills (1987) found an intermediate level of enzyme activity in ADH heterozygotes of $S$. cerevisiae, while at a more "integrated" phenotypic level, overdominance for clone diameter was observed in the ADH heterozygotes. That true overdominance can result from additive gene action was noted already long ago (Crow 1952). A non-linear mapping between gene action and phenotype is necessary for that purpose.

Some clarification is needed at this point on the use of the terms overdominance and heterosis in this paper. Overdominance is often used to indicate a fitness pattern with heterozygote advantage, especially when a perfect correlation between phenotype and viability fitness is assumed. In this paper, the term overdominance applies to phenotypes only. Individuals that are heterozygous at a specific locus are overdominant when their phenotypes are (on average) larger in size than the phenotypes of individuals homozygous for the respective alleles. Heterosis (Schull 1914) originally denoted the hybrid vigour that often occurs when crossing individuals from different inbred lines. The use 
of the term heterosis to indicate a fitness advantage for heterozygotes, started with Dobzhansky (Dobzhansky 1952). Dobshansky made a distinction between euheterosis due to inbreeding depression and balanced heterosis, which is the result of phenotypic overdominance and selection. He believed that balanced heterosis is rather exceptional: mutations for overdominant mutant heterozygotes should occur with low probability in comparison with mutational effects giving partially dominant heterozygotes (Dobzhansky 1952). However, natural selection can be particularly effective at sieving out such rare mutants.

I propose that evolution is at least part of the reason for the rare occurrence of overdominant heterozygous phenotypes. An evolutionary model is used to argue this point. In the argument developed, the use of population genetic or genotypic fitness (Maynard Smith 1998) is avoided. Instead, invasion fitness is used which is a long-term fitness measure (Metz et al. 1992). It can be defined for any faithfully reproducing entity, such as clonally reproducing phenotypes, or alleles in a sexual and diploid setting. Invasibility conditions are an important tool in population genetics (Fisher 1930), and invasion fitness allows for an extension of the well-known population genetical toolbox to more realistic ecological scenarios with population structure and both stochastic as well as density-dependent contributions to the population dynamics (Metz et al. 1992; Ferrière and Gatto 1995). Invasion fitness has been used already in studies that address the evolution of the genetic system (Kisdi and Geritz 1999; Van Dooren 1999; Kisdi and Geritz in press).

In this paper, I construct and analyse a long-term evolutionary model that allows for phenotypically overdominant heterozygotes. A model example of body size evolution is presented instead of a more general argument leading to the same conclusions (Van Dooren 2000), for three reasons. (i) Many empirical studies have tried to distinguish between size effects from direct phenotypic overdominance and effects following from the presence of deleterious alleles (e.g., Strauss 1986; Zouros et al. 1988; Houle 1989; David et al. 1995; Savolainen and Hedrick 1995; Xiao et al. 1995; Bierne et al. 1998; Pogson and Fevolden 1998). (ii) The model and the results can be used to implement and organize individual-based simulations. (iii) By means of Pairwise Invasibility Plots (Van Tienderen and de Jong 1986; Kisdi and Meszéna 1992; Metz et al. 1992, 1996), properties of this evolutionary dynamical system with diploid and sexual inheritance can be compared with an equivalent system that has clonal inheritance.

The model is hierarchically structured. A phenotype recipe mapping allelic traits to individual body size is embedded into a population ecological scenario to which individuals are subjected. A single-locus model for body size evolution is developed first. The effects from other loci contributing to adult body size are brushed into a number of genetic background parameters. Using this model example, the conditions are investigated that favour evolution towards a population state where overdominance can be ob- 
served. I subsequently discuss the evolution of overdominance in a multi-locus context, where both the focal locus and the genetic background evolve. The main conclusion is that, overall, direct phenotypic overdominance is expected to be rare.

\section{A Phenotype Recipe for Body Size}

In this section, a phenotype recipe for body size is constructed that incorporates mechanisms assumed to cause overdominance (Crow 1952, Hull 1952). Direct overdominance might not only be rare or has been hard to demonstrate in practise (Lynch and Walsh 1998), many studies do not aim further than a simple demonstration of its presence. The probability of occurrence of different presumed causative mechanisms is not investigated. Next to serving its purpose in this evolutionary model, a phenotype recipe incorporating such a mechanism can be used as a starting point for that kind of investigation. I tried to achieve a simple genotype-phenotype map that has the required properties in order to produce overdominant heterozygotes. It contains elements that are standard models for certain processes. The phenotype recipe incorporates an expression for metabolic flux from metabolic control theory (Kacser and Burns 1973) and uses a simple model for body size growth (von Bertalanffy 1934).

The amount of energy that is available for individual growth depends on the profit from metabolic pathways that convert resources into energy-equivalent growth units (Koehn 1991). In calculating growth units or metabolic profit from a pathway, we have to consider both the benefit from the pathway flux as well as the cost involved in maintaining the functional enzyme pools in the pathway. Maximum metabolic profit occurs when the difference between metabolic benefit and cost is the largest possible. The benefit of a pathway or the pathway flux will show an increase in flux with increasing enzyme activity, but also a diminishing return (Kacser and Burns 1973). Enzyme activities are partly determined by enzyme steady state concentrations, such that the maintenance cost of the pathway will also increase with the steady state concentrations (Brown 1991). A larger flux through the pathway effected by a change in enzyme structure might as well imply an additional cost in transporting the reaction products against a concentration gradient, or such a change might require additional protection to prevent the changed enzyme from degrading. Thus, both metabolic costs and benefits will generally increase with enzyme activity. Since benefits will hardly outweigh costs for very small levels of activity and since costs will be much larger than benefits for an almost infinite amount of enzyme activity, maximum metabolic profit will most often occur at intermediate values of total enzyme activity from a locus.

Based on simple physiological considerations, one can easily construct a phenotype recipe for adult body size with maximum metabolic profit at intermediate total enzyme activity. Body size is assumed to derive from enzyme activities of gene products in two 
steps: total enzyme activity per locus determines natal or initial growth rate, which in turn determines adult size. I assume additivity of effects at the level of enzyme activity: metabolic profit is a function of the total activity from the locus. This is a reasonable assumption for metabolic housekeeping loci (Kacser and Burns 1981, Van Dooren 1999).

The following non-linear function is a relatively simple expression for the metabolic profit emerging from two allele activity parameters at one locus in a pathway (Eqn. 1). I assume that natal growth rate is proportional to this metabolic profit.

$$
\phi_{1}\left(x_{1}, x_{2}\right)=\frac{c_{1}\left(x_{1}+x_{2}\right)}{1+c_{2}\left(x_{1}+x_{2}\right)}-c_{3}\left(x_{1}+x_{2}\right)^{2}-c_{4}
$$

Allelic are characterized by parameters $x_{1}$ and $x_{2}$ that denote the enzyme activities of the gene products of alleles 1 and 2 as far as these are controlled by the locus itself. They are allowed to vary over the positive real numbers, and it is assumed that zero or negative metabolic profit will imply zero body size. The trait space in which evolution takes place is therefore limited to the allele parameters that result in noticeable body sizes. The first term of Equation (1) represents the benefit from the pathway flux in terms of enzyme activity (Kacser and Burns 1973). It is an extremely simple expression that satisfies the most important requirements for an expression of flux: zero activity implies no flux, and an infinite flux is impossible. Second and third terms in Equation (1) are pathway maintenance costs. I opted for a quadratic cost function of the total enzyme activity per locus. This is a harmless assumption in terms of the conclusions and it ensures that phenotype recipes with multiple metabolic profit loci can show the same evolutionary behaviour as this single-locus model (Van Dooren manuscript). For simplicity, metabolic profit does not depend on an explicit environmental parameter.

Parameters $c_{i}$ stand for contributions of the genetic background to metabolic profit. These parameters summarise the effects of alleles present at other loci involved in constructing the phenotype. Loci in the genetic background can have different linear or non-linear relationships between allele parameters and metabolic profit, and each locus is allowed to affect several genetic background parameters at once. It is assumed that the genetic background is homogeneous, i.e., there is one allele at each locus contributing to genetic background effects.

Loci with regulating effects that modify the enzyme activities of other loci are ubiquitous. Assuming that the total gene action from such modifying loci has a multiplicative effect on total enzyme activity from the focal locus, the effects of regulating loci on the activity at the focal locus can be included in the background parameters $c_{1}, c_{2}$ and $c_{3}$. The allelic trait parameters at the focal locus therefore represent the contribution to ac- 
tivity from the locus itself, with the effects of external regulation factored out.

For mapping natal growth rate to body size, one can assume von Bertalanffy growth (von Bertalanffy 1934). In that case, final size (measured as body length) is proportional to natal growth rate and hence metabolic profit (Eqn. 2, Metz and Diekmann 1986b). Parameter $\gamma$ scales body size to metabolic profit.

$$
\phi_{2}\left(\phi_{1}\right)=\gamma \phi_{1}
$$

Combining maps (1) and (2) allows us to write the phenotype recipe for body size as a function of the allele activity parameters (Eqn. 3)

$$
\phi\left(x_{1}, x_{2}\right)=\phi_{2}\left(\phi_{1}\left(x_{1}, x_{2}\right)\right)
$$

Maximum body size can be realised by one homozygous genotype as well as infinitely many heterozygotes, since additivity occurs at the level of enzyme activity. Pairs of alleles with total activities in homozygotes on both sides of the total activity corresponding to maximum body size can have overdominant heterozygotes (Fig. 1).

For pairs of alleles with homozygote activities that are both on either side of that total activity, intermediate dominance is found. It is the pronounced non-linearity and the existence of an intermediate maximum in the mapping between total activity and body size that allows for different possible dominance interactions, ranging from nearly additive genetics until overdominance. One can call loci with a maximum phenotypic effect for intermediate total activities metabolic profit loci.

\section{Population Dynamics}

At the population ecological level, a Lotka-Volterra competition model is assumed with distinct zygote formation and gamete production episodes (Christiansen and Loeschke 1980; Hofbauer et al. 1987; Rand et al. 1994; Ferrière and Cazelles 1998). When there is only one allele in the population, the mean field equation of the populatrion dynamics becomes the well-known Ricker equation (Ricker 1954). Number of offspring then has a simple exponential dependence on population density, which is a type of density dependent function often fitted to ecological time series (Turchin 1995). The population 


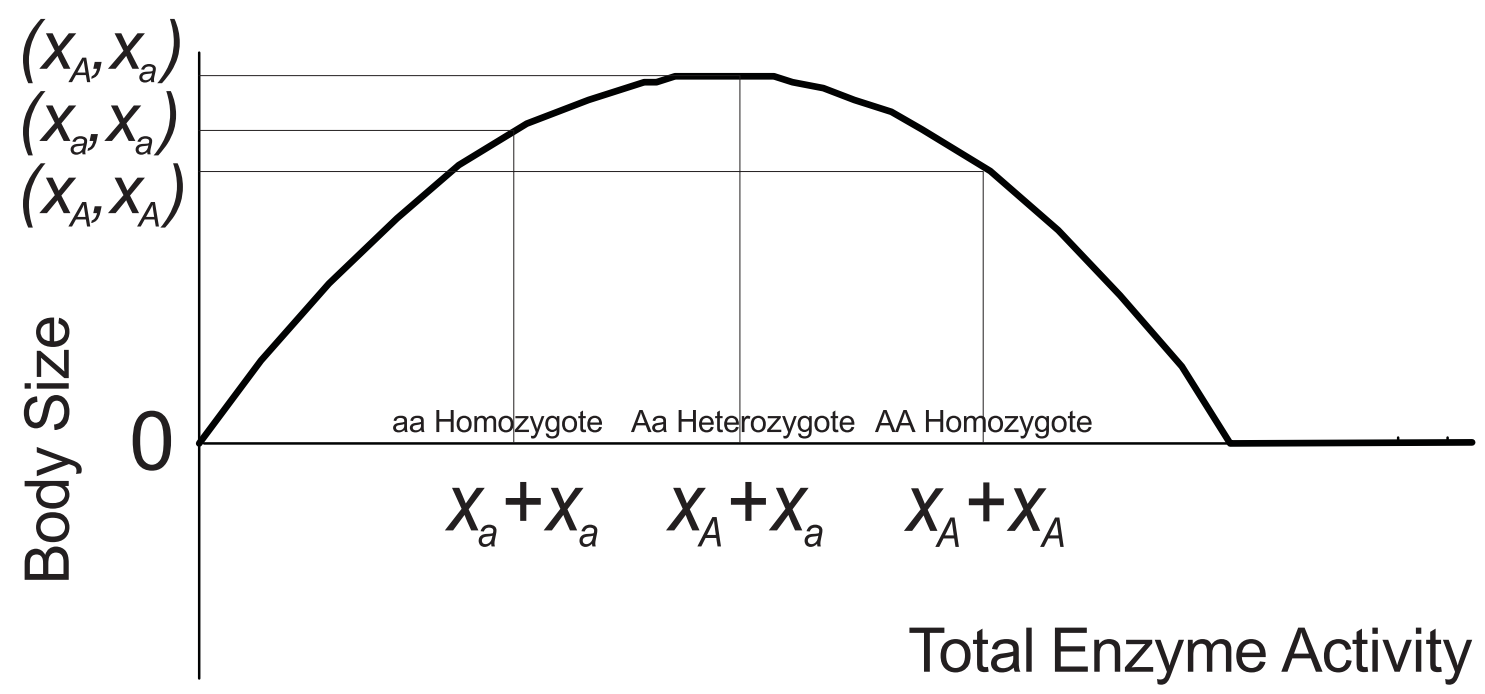

Figure 1 This figure shows body size as a function of total enzyme activity at a metabolic profit locus. Overdominance for body size occurs when body size has a maximum value at intermediate values of total enzyme activity. Additivity is assumed at the level of total enzyme activity. For a pair of alleles with different enzyme activities $x_{A}$ and $x_{a}$ of gene products, total enzyme activities and body sizes of homozygotes and heterozygote are indicated. The alleles $A$ and $a$ have a heterozygote with overdominant body size.

ecological scenario has the additional advantage that mean field equations can be derived from explicit individual-based arguments (Royama 1992, Leitner 1998). Such explicitness is highly advantageous. Individual-based simulations become transparent and easy to implement (see Van Dooren, manuscript), the interpretation of results often becomes easier and it is straightforward to modify an explicit model on the basis of results from specific experiments. This section describes the ecological scenario and gives mean field models for the dynamics of a population with one common or resident allele and for the dynamics of a rare mutant allele appearing in such a population. In Appen$\operatorname{dix} \mathrm{A}$, the derivation of the mean field equations from individual-based arguments is given for this genetically explicit model and allowing for genetic polymorphism.

In the Lotka-Volterra competition system assumed, individuals live in different patches where reproduction occurs [This makes it easier to set up simulations than with interaction neighbourhoods of a fixed size as in Royama (1992) and produces the same mean field model]. After random mating of gametes in a common pool, zygotes or individuals settle at random in one of the patches. Reproduction or gamete production depends on the number of individuals present on the patch and on their phenotypes. Survival of individuals between reproduction episodes is zero. Parameter $b_{\max }$ is the average number of offspring gametes from a parental individual that enter the next reproduction episode, when the parent was alone on the patch and did not experience competition. In this Lotka-Volterra competition model, the actual number of gametes entering the reproduction episode in the next generation equals $b_{\max }$ multiplied by the competition effects from all other individuals present on the patch (Royama 1992). Each indi- 
vidual also present on the patch decreases the number of gametes. Parameters $\delta_{i j, k l}$ represent the multiplicative adjustment of the reproduction of an individual with phenotype $\phi\left(x_{i}, x_{j}\right)$ by an individual of phenotype $\phi\left(x_{k}, x_{l}\right)$ also present on the same patch. This parameter is a factor between zero and one, by which each additional individual decreases reproduction. One minus this quantity can be interpreted as a measure of competition intensity from one phenotype upon another.

In the mean field model for a population with only one common or resident allele, the average number per patch (the local density) $X_{r, t}$ of resident allele $x_{r}$ changes over time according the Ricker equation (Ricker 1954). The recurrence equation for this population composition is given by Equation 4 .

$$
X_{r, t+1}=X_{r, t} \frac{b_{\max }}{2} e^{-\frac{\left(1-\delta_{r r, r}\right) X_{r}}{2}}
$$

The number of alleles entering the next generation depends on the maximum average number of gametes $b_{\max }$, on the local density of resident alleles $X_{r, t}$ and on the competition intensity between resident individuals $\left(1-\delta_{r r, r r}\right)$. The population dynamics of this Ricker system (Eqn. 4) can have a stable equilibrium density or a cyclic or chaotic attractor depending on the value of the fecundity parameter $b_{\max }$.

A mutant allele $x_{m}$ is initially rare in comparison to the resident allele $x_{r}$. It approximately occurs in heterozygous individuals $\phi\left(x_{m}, x_{r}\right)$ of mutant and resident alleles only. The repercussion of the local density of individuals with a mutant allele on the dynamics of the resident allele and on the reproduction of other mutant individuals can be considered negligible. Equation (5) gives the expression for the mean field population dynamics of a rare mutant allele $x_{m}$ with local density $X_{m, t}$, when this mutant appears in a resident population system with allele $x_{r}$ (and local density $X_{r, t}$ ).

$$
X_{m, t+1}=X_{m, t} \frac{b_{\max }}{2} e^{-\left(1-\delta_{m r, m r}\right) \alpha_{m r, r r} \frac{X_{r, t}}{2}} \text { with } \alpha_{m r, r r}=\frac{1-\delta_{m r, r r}}{1-\delta_{m r, m r}}
$$

Because of initial rarity of mutants, the density of mutant alleles is omitted from the density-dependent multiplication factor. In Equation 5, a function $\alpha_{m r, r r}$ is introduced. It is the ratio of the competition intensity from the resident phenotype $\phi\left(x_{r}, x_{r}\right)$ upon the mutant phenotype $\phi\left(x_{m}, x_{r}\right)$, and the intensity of competition between individuals of the mutant phenotype. In Eqn. (5), the local resident population density is multiplied by a factor $\left(1-\delta_{m r, m r}\right)$ that represents competition as it should occur between mutant phenotypes and a factor $\alpha_{m r, r r}$ that scales the competition effect from resident phenotype upon 
mutant phenotype relative to the first factor (see App. A).

Assuming that competition between different types is always less than between identical ones, function $\alpha$ will take on values between zero and one. For two (resident) individuals with identical phenotypes, it equals one. With increasing difference between, for instance, a mutant $\phi\left(x_{m}, x_{r}\right)$ and a resident phenotype $\phi\left(x_{r}, x_{r}\right)$, the value of the ratio $\alpha_{m r, r r}$ is assumed to decrease towards zero.

In order to calculate competition effects between various phenotypes indexed $i j$ and $k l$, i.e., with genotypes $\left(x_{i}, x_{j}\right)$ and $\left(x_{k}, x_{l}\right)$, functions $\delta_{i j, k l}$ need to be specified. This is done indirectly by defining functions for $\alpha_{i j, k l}$ and $\delta_{i j, i j}$, from which competition effects $\delta_{i j, k l}$ can be calculated as $\delta_{i j, k l}=1-\alpha_{i j, k l}\left(1-\delta_{i j, i j}\right)$. In this example, the function $\alpha_{i j, k l}$ for individuals indexed $i j$ and $k l$, is modelled by means of a bell-shaped curve (Eqn. 6).

$$
\alpha_{i j, k l}=e^{-v\left[\phi\left(x_{i}, x_{j}\right)-\phi\left(x_{k}, x_{l}\right)\right]^{2}}
$$

Competition parameter $v$ controls how fast competition levels off with increasing difference between individuals. Competition between different types levels off faster with increasing $v$.

Function $\delta_{i j, i j}$ needs to have a value between zero and a maximum value smaller than one. With a maximum value of one, identical types would not affect each others reproduction. One can assume that there is an intermediate body size for which $\delta_{i j, i j}$ is maximal with value $s$ (competition intensity then is minimal), and that it decreases to zero with increasing difference from that body size phenotype. For $\delta_{i j, i j}$ I chose the quadratic function

$$
\delta_{i j, i j}=\left[s \phi\left(x_{i}, x_{j}\right)\left(2-\phi\left(x_{i}, x_{j}\right)\right)\right]_{+} \text {with } 0<1<s
$$

This effect of an individual on the growth rate of individuals with the same phenotype/genotype (Eqn. 7), mimicks the competition effect of a resource which is limiting and affects reproduction in a phenotype-dependent manner. Conditions on parameter $s$ ensure that $\delta_{i j, i j}$ has a maximum value between zero and one. The maximum value of $\delta_{i j, i j}$, occurs when the body size of the phenotype indexed $i j$ equals one. Any range of body sizes occurring in the model can be adjusted or scaled in order to fulfil this condition. Individuals with body size 1 produce $b_{\max } \delta_{i j, i j}=b_{\max } s$ offspring gametes when they have one other neighbour on the patch with identical phenotype [This is derived from individual-based arguments, not from the mean field model Eqn. (4)]. Individuals with other phenotypes produce fewer gametes in the presence of one identical neighbour, be- 
cause they compete more for a limiting resource. A bell-shaped curve could be used for $\delta_{i j, i j}$ as well, but I decided to approximate it by a parabola because it has one parameter less than a similar Gaussian, and because both functions have approximately the same shape around their maximum. This approximation does not affect the evolutionary dynamics (Van Dooren, unpublished results). The function in Eqn. (7) represents scramble competition that is sometimes extreme. When $\delta_{i j, i j}=0$, the presence of only two identical individuals $i j$ on the patch is already fatal for their reproduction. When the two individuals are slightly different, they again have a chance to reproduce succesfully. Note that this formulation of competition can imply $\delta_{i j, k l} \neq \delta_{k l, i j}$ while $\alpha_{i j, k l}=\alpha_{k l, i j}$.

\section{Evolutionary Dynamics}

In this section expressions are given for the invasion fitness of alleles and phenotypes. I will show under which conditions an evolutionary random walk goes towards the homozygote with the total enzyme activity that gives a maximum value of body size

\section{Invasion Fitness}

Invasion fitness (Metz et al. 1992, Rand et al. 1994, Ferrière and Gatto 1995) is a fitness currency that can be used in a broader range of ecological scenarios that the notion of viability fitness often used in population genetics. Invasion fitness is the long-term average growth rate of a population of mutant alleles in a persistent resident population dynamical system (Eqn. 8), where it is assumed that the mutant population density $X_{m, t}$ is negligible in comparison with population densities of resident alleles.

$$
\zeta\left(x_{m}, x_{r_{1}}, \ldots, x_{r_{n-1}}\right)=\lim _{t \rightarrow \infty} \frac{1}{t} \ln \frac{X_{m, t}}{X_{m, 0}}
$$

Invasion fitness $\zeta$ (Eqn. 8) is a function of the enzyme activity trait of the mutant allele $x_{m}$, and the traits $x_{r_{i}}$ of the $n$-1 different resident allele types $(i=1, \ldots, n-1)$. It is always zero when the mutant allelic trait is equal to a resident allelic trait parameter. Invasion fitness is the key mathematical tool in evolutionary and adaptive dynamics, which are both dynamic extensions of the traditional ESS toolbox (Maynard Smith 1982; Metz et al. 1996; Dieckmann 1997; Diekmann 1997). When ecological and evolutionary timescales are different, the evolutionary process becomes mutation-limited. In that case, the process of evolution can be described as a directed random walk over the possible resident population states (Dieckmann and Law 1996; Metz et al. 1996). Invasion fitness is then used to determine which transitions between resident states are possible (Metz et al. 1996). The probability of invasion of a rare mutant allele can be estimated from in- 
vasion fitness (Ewens 1969; Athreya and Karlin 1971; Haccou and Iwasa 1996). Only when invasion fitness is positive, the mutant has a positive probability of invasion.

In this section, the study of the evolutionary dynamics of the model example uses an invasion fitness expression for mutant alleles in populations of one resident allele only (Eqn. 9). It can be derived from the mean field models for the popuylation dynamics of mutant and resident alleles, Eqns. (4) and (5). The derivation is given in Appendix B. Invasion fitness (Eqn. 9) has the prescriptions for competition functions $\alpha$ and $\delta$ alreay inserted.

$$
\zeta\left(x_{m}, x_{r}\right)=\ln \frac{b_{\max }}{2}\left(1-e^{-v\left(\phi_{M}-\phi_{R}\right)^{2}} \frac{1-\left[s \phi_{M}\left(2-\phi_{M}\right)\right]_{+}}{1-\left[s \phi_{R}\left(2-\phi_{R}\right)\right]_{+}}\right)
$$

In this expression for the invasion fitness of a mutant allele (Eqn. 9), $\phi_{M}$ denotes the heterozygous phenotype of the mutant individuals $\phi_{M}=\phi\left(x_{m}, x_{r}\right)$, and $\phi_{R}$ the phenotype of the resident homozygous individuals $\phi_{R}=\phi\left(x_{r}, x_{r}\right)$. Invasion fitness is, also for this genetic-ecological model, first of all a function of demographic parameters. These demographic parameters are determined from function prescriptions that have mutant and resident phenotypes as arguments, which are in turn functions -phenotype recipesof mutant and resident allele parameters.

The population dynamics of the resident alleles (Eqn. 4), the mutant alleles (Eqn. 5) and invasion fitness (Eqn. 9) in this system, can as well be read as equations from a model with one mutant phenotype, one resident phenotype and with clonal inheritance. If we substitute the number of resident individuals for alleles, $2 N_{r r, t}=X_{r, t}$, Equation (4) can be transformed into an equation for the dynamics of resident individuals. The number of mutant alleles $X_{m, t}$ equals the number of mutant individuals $N_{m r, t}$ and the amount of resident alleles produced by mutant individuals is negligible. Equations (4) and (5) do not change if we assume individuals to reproduce clonally. Clonal inheritance and reproduction is an assumption often made in adaptive dynamics models (Dieckmann 1997). With this assumption, the contribution of the population ecology to the evolutionary dynamics can be singled out. Modification of Eqns. (4) and (5) into mean field models for densities of individuals and assuming clonal inheritance gives a completely phenotypic model, with an invasion fitness expression equal to Equation (9). Expression (9) thus also gives the invasion fitness for a pair of clonally reproducing mutant $\phi_{M}$ and resident $\phi_{R}$ phenotypes, assuming the same population ecology as explained in the previous section, but with individuals producing individuals and no mating in the common pool. We can express this double interpretation of invasion fitness by the equality (10), 


$$
\zeta\left(x_{m}, x_{r}\right)=\sigma\left(\phi_{M}, \phi_{R}\right)
$$

where $\sigma$ denotes invasion fitness in the clonal or phenotypic interpretation. In this case of invasion fitness in a resident population of one type of individual and allele, Equation (10) implies that the evolutionary effects of adding Mendelian single-locus genetics onto a phenotypic evolutionary model, without changing the population ecology, will depend on the phenotype recipe assumed, i.e., on the way allele parameters translate into phenotypes (see also Geritz et al. 1998; Kisdi and Geritz 1999; Van Dooren in press).

\section{Evolutionary Attractors}

Evolutionary dynamical systems have an evolutionary attractor (EA) or several ones, just as a population dynamical system has one or several population dynamical attractors. Evolutionary attractors are population states that can be found through forward iteration of the evolutionary dynamical system [this follows an informal definition of attractor as in Collet and Eckmann (1980), for instance] and which are stationary on an evolutionary timescale, provided that the population ecology does not change. They are sets of alleles that are evolutionarily attracting and that cannot be invaded by mutant alleles [evolutionary attractivity and invasibility are explained below, strictly speaking the EA considered are evolutionary stable fixed point attractors].

We can assume that an evolving population system initially contains one common allele, and then find the evolutionary attractors of the system using the approach of Evolutionary Random Walks or Adaptive Dynamics (Dieckmann and Law 1996; Metz et al. 1996; Geritz et al. 1998). In this approach, mutation rates at all the loci are decreased such that evolution becomes mutation limited and can be described as an evolutionary random walk. Invasion fitnesses and invasion fitness gradients are then sufficient to determine which resident population states are EA's. First of all, it is determined whether evolution will halt at a population state with one allele, or whether it will produce populations with two or more very different alleles.

\section{Two Types of Candidate ESS}

Evolutionary attractors of one allele or phenotype are also called Continuously Stable Strategies (Eshel 1983). These are Evolutionarily Stable Strategies (ESS; Maynard Smith 1982) that are also evolutionarily attracting. CSS's and points in trait space where transitions occur from one resident allele to two resident alleles (these are a class of socalled evolutionary branching points; Metz et al. 1996) are found at points in trait space where the partial derivative of invasion fitness (Eqn. 9) with respect to the mutant trait parameter is zero, when evaluated for the mutant trait equal to the resident trait pa- 
rameter. These allele or strategy parameters are often called candidate ESS strategies and denoted $x^{*}$. Evaluating where exactly in trait space this partial derivative becomes equal to zero, is the standard way of locating candidate ESS strategies. Candidate ESS's are also called monomorphic evolutionarily singular strategies (Metz et al. 1996) since the gradient of invasion fitness is zero at these points. I will use the abbreviation cESS for such points.

Invasion fitness (9) is a function of demographic parameters. These depend on phenotypes, which in turn depend on allele parameters. If we take a partial derivative of invasion fitness (Eqn. 9), we can use the chain rule of derivatives as an aid in clarifying what exactly in the expression will make it become zero. In the context of this genetic model, it is most insightful to take the derivative with respect to the mutant phenotype first, and then multiply it with the derivative of the mutant phenotype recipe with respect to the mutant allele parameter (Eqn. 11).

$$
D_{x_{m}} \zeta\left(x_{m}, x_{r}\right)=D_{\phi_{M}} \sigma\left(\phi_{M}, \phi_{R}\right) . D_{x_{m}} \phi_{M}
$$

The first factor in Equation (11) is the derivative of invasion fitness with repect to the mutant phenotype. It depends on the population ecology. In order to make it clear that this derivative depends on the population ecology of phenotypes only, one can write it as a derivative of the clonal/phenotypic interpretation of invasion fitness $D_{\phi_{M}} \sigma$. The second factor $D_{x_{m}} \phi_{M}$ is the derivative of the mutant phenotype recipe with respect to the mutant allele activity. This factor depends on the genetics and not on the population ecology.

In the trait space of allelic activity parameters, candidate ESS's $x^{*}$ are located at points where the derivative $D_{x_{m}} \zeta\left(x^{*}, x^{*}\right)$ becomes zero. That occurs when either of the two factors $D_{\phi_{M}} \sigma$ or $D_{x_{m}} \phi_{M}$, evaluated for a mutant (allelic or phenotypic) trait parameter equal to the resident trait parameter, becomes equal to zero (Eqn. 12).

$$
D_{x_{m}} \zeta\left(x^{*}, x^{*}\right)=0 \Leftrightarrow D_{\phi_{M}} \sigma\left(\phi^{*}, \phi^{*}\right)=0 \text { or } D_{x_{m}} \phi\left(x^{*}, x^{*}\right)=0
$$

A candidate ESS phenotype $\phi^{*}=\phi\left(x^{*}, x^{*}\right)$ where clonal invasion fitness $\sigma$ is at a local extremum with respect to the mutant phenotype, can be called 'population' or $P$-level cESS because it arises from the population ecology. cESS's or singular points $x^{*}$ following from a local extremum of the phenotype recipe $\phi$ are called 'individual' or $I$ level singular points, since interactions between genes within individuals generate them. Candidate ESS allelic activity parameters $x^{*}$ thus fall into two categories, denoted $x^{P}$ and $x^{I}$, for P- and I-level candidate ESS's respectively. 
The phenotypes for which clonal invasion fitness has a local extremum are homozygous phenotypes $\phi^{P}=\phi\left(x^{P}, x^{P}\right)$ of P-level cESS allele activity parameters. Evaluating the partial derivative of 'phenotypic' invasion fitness $D_{\phi_{M}} \sigma$, one finds that there is only one cESS body size in this model for which the partial derivative, evaluated at equal mutant and resident phenotypes, becomes zero. It equals one: $\phi^{P}=1$. When this body size is within the range of the phenotype recipe, it immediately follows from the shape of the phenotype recipe that two different alleles will produce the candidate ESS body size, unless the cESS coincides exactly with the maximum body size of the phenotype recipe. Therefore, for one P-level cESS phenotype $\phi^{P}$, there will be almost surely two cESS allele activities $x_{1}^{P}$ and $x_{2}^{P}$ with $\phi^{P}=\phi\left(x_{1}^{P}, x_{1}^{P}\right)=\phi\left(x_{2}^{P}, x_{2}^{P}\right)=1$.

In this model, one I-level cESS occurs for the allele activity parameter $x^{I}$ that produces maximum body size in homozygous individuals. The homozygous phenotype of the I-level cESS allele $x^{I}$ can be written as $\phi^{I}$. The allele $x^{I}$ generally will not produce a local extremum of the clonal invasion fitness expression as well. When a phenotype recipe is not of metabolic profit type and has no local maximum, then it will have intermediate genetics always. Overdominant heterozygotes will not be possible, which implies that the only cESS's in the system will be the ones generated by the population ecology.

\section{Pairwise Invasibility Plots}

In order to visualize the pattern of invasion fitnesses for different combinations of resident and mutant trait parameters, Pairwise Invasibility Plots (PIP's; Van Tienderen and de Jong 1986; Kisdi and Meszéna 1992; Metz et al. 1992, 1996) are very useful. On such a plot one finds the same trait parameter on both axes. Every point on it represents a combination of mutant (vertical axis) and resident trait values (horizontal axis). A code indicates whether, for a given evolutionary model, the mutant with that specific trait value can invade a population with the resident trait value or type specified on the horizontal axis. There are two related PIPs that one can make for the model in this paper, a clonal one and a diploid genetic one (Figure 2; Van Dooren in press). In the clonal PIP, one puts resident and mutant phenotypes on the axes. In the diploid PIP, the resident and mutant allelic trait parameters -in this case enzyme activities of allele gene products- are on the axes. The range of the clonal PIP in Fig. 2 goes from slightly below the cESS phenotype $\phi^{P}$ until the maximum body size $\phi^{I}$. The diploid PIP ranges over all allelic activity parameters that produce non-zero body sizes. Invasion fitness (Eqn. 9) for a pair of alleles is calculated as the invasion fitness of the mutant and resident phenotypes. Any pair of resident and mutant allelic parameters $x_{r}$ and $x_{m}$ in the diploid PIP correspond to a pair of resident and mutant phenotypes $\phi_{R}=\phi\left(x_{r}, x_{r}\right)$ and $\phi_{M}=\phi\left(x_{m}, x_{r}\right)$ that are used to calculate invasion fitness. As a consequence, points $\left(x_{r}, x_{m}\right)$ in the dip- 


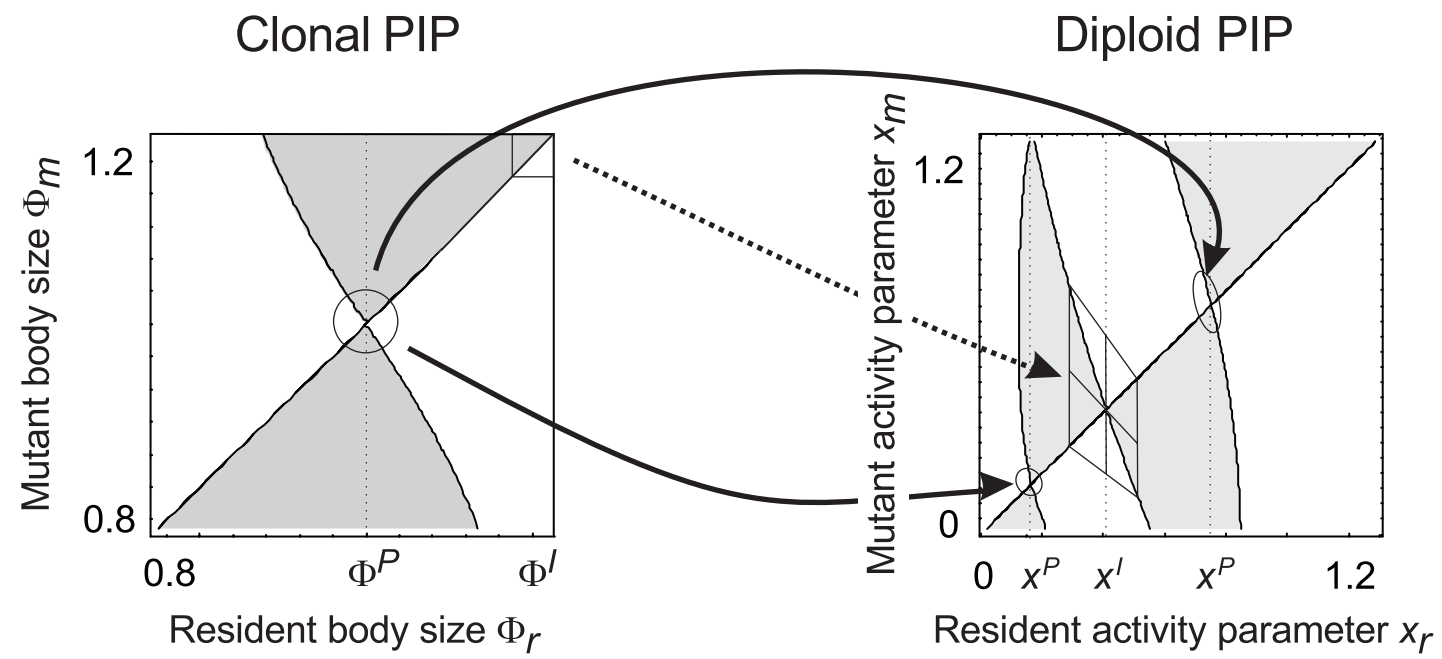

Figure 2 The relationship between clonal and diploid Pairwise Invasibility Plots is clarified. The population ecological scenario is identical in both plots $\left(b_{\max }=12, v=2, s=0.9\right)$. This ecological scenario has a globally attracting ESS body size $\phi^{P}$. In diploid evolutionary models where overdominance can occur, candidate ESS's either arise from the population ecology $(\mathrm{P})$ or from the genotype-phenotype map (I). In the diploid PIP, the invasion fitness pattern in a neighbourhood around a P-level cESS allele $x^{P}{ }_{i}$ is identical to the pattern around the global ESS $\phi^{P}$ in the clonal PIP (connected and encircled). The genotypephenotype map $\left(c_{1}=6, c_{2}=4, c_{3}=0.4, c_{4}=0\right.$ and $\left.\gamma=1.2\right)$ produces that body size for two values of total enzyme activity $x_{1}^{P}$ and $x_{2}^{P}$, hence two different resident alleles are evolutionary attractors. At the enzyme activity giving maximum body size in homozygotes, an invasible and evolutionarily repelling candidate ESS $x^{I}$ can be observed. The pattern of invasion fitness around it derives from the boxed neighbourhood of the I-level cESS phenotype $\phi^{I}$ of the clonal PIP in a non-trivial way. This neighbourhood is repeated four times on the diploid PIP. It also appears mirrored in the horizontal and/or the vertical direction.

loid Pairwise Invasibility Plot can be mapped to points $\left(\phi_{R}, \phi_{M}\right)$ in the clonal PIP with equal invasion fitness, and vice versa (Fig. 2; Van Dooren in press). The effect of a specific phenotype recipe on the pattern of invasion fitness can be investigated through this mapping, by comparing the pattern of invasion fitnesses in the clonal and the diploid PIP. The pattern of invasion fitnesses on the clonal PIP depends on the population ecology only. In the diploid plot it depends on the population ecology and on the phenotype recipe.

In each type of PIP, candidate ESS's occur where the sign pattern of invasion fitness changes across the 45 degree line. These cESS are indicated in Figure 2. The phenotypic cESS $\phi^{P}=1$ on the clonal PIP corresponds to a pair of allelic cESS's on the diploid PIP. There are two such candidate ESS's because the specific phenotype recipe used to construct the plot returns that specific body size for two values of total enzyme activity. The I-level cESS occurs, for the parameters used to produce Figure 2, at the allele activity parameter $x^{I}=0.4$. This allele activity corresponds to a homozygous maximum body size with value $\phi^{I}=1.22$. On the same figure, one can see that the mapping between the clonal and diploid PIP's is not always a simple deformation changing the shape of the 
boundary of each specific region of resident-mutant combinations a bit when going from one plot to the other. The region of invasion fitness indicated as a boxed neighbourhood on the clonal PIP, is repeated four times in the neighbourhood around the diploid I-level cESS. It also appears mirrored in the horizontal and/or the vertical direction. That happens because phenotypes slightly smaller than the maximum possible body size occur for allele activity parameters both smaller and larger than the cESS allelic parameter $x^{I}$

\section{Evolutionary Attractivity and Invasibility}

Whether the evolutionary dynamics will approach a candidate cESS, depends on its evolutionary attractivity. Once arrived there, the invasibility of the cESS decides whether evolution will halt at the cESS not. To determine evolutionary attractivity and invasibility of cESS's, second derivatives of invasion fitness need to be evaluated (Metz et al. 1996; Geritz et al. 1997, 1998). One can also read the invasibility and evolutionary attractivity of each cESS on a PIP (see Dieckmann (1997) for a nicely represented overview of possible patterns).

A ESS allele activity parameter $x^{*}$ is evolutionarily attracting when $D_{r r} \zeta\left(x^{*}, x^{*}\right)-D_{m m} \zeta\left(x^{*}, x^{*}\right)>0$, with $D_{i j} \zeta$ denoting the second derivative of diploid invasion fitness with respect to arguments $x_{i}$ and $x_{j}$. Attractivity means that a resident strategy similar to the candidate ESS can be invaded by a mutant type even more similar to the cESS (Eshel 1983; Christiansen 1991). On PIP's such as in Figure 2, local attractivity of a cESS is determined from the sign pattern in a small neighbourhood of the 45 degree line. A candidate ESS is attractive when an allele with a trait parameter similar to the cESS trait value can be invaded by alleles that are slightly more similar to the cESS. In that case, when crossing the 45 degree line at the left of the cESS and in the direction of increasing trait values, the sign changes from negative to positive. On Fig. 2 , the cESS phenotype $\phi^{P}$ and the two P-level cESS's allele parameters $x_{1}^{P}, x_{2}^{P}$ are evolutionarily attracting, the I-level cESS $x^{I}$ is not.

A cESS $x^{*}$ is not invasible or unbeatable (Eshel 1996; Metz et al. 1996) when $D_{m m} \zeta\left(x^{*}, x^{*}\right)<0$. On a PIP, invasibility is determined from the sign pattern at a cESS $x^{*}$ in the vertical direction across the 45 degree line. In Fig. 2, a vertical line drawn through the P-level cESS allele parameters goes, near to the 45 degree line, through a region with negative values of invasion fitness. That means that these P-level cESS's, as a resident allele, can not be invaded by alleles with similar activity parameters. The Ilevel cESS allele is invasible by all slightly different alleles.

A cESS $x^{*}$ that is both evolutionarily attracting and not invasible is a Continuously Stable Strategy, or an evolutionarily attracting ESS. It represents an endpoint of the evolutionary process, an evolutionary stop. Evolutionary branching occurs when the 
population is near to a cESS that is both evolutionarily attracting and invasible (Metz et al. 1996; Geritz et al. 1998). The evolutionary random walk then is first attracted towards the cESS. Once there, the cESS can be invaded by alleles with both smaller and larger activity parameters. The initial population with one resident allele will be replaced by a population with two alleles, having parameters both smaller and larger than the cESS value. This is evolutionary branching. Selection will keep both alleles in the population in a protected polymorphism (Prout 1968) because each allele can be shown to increase in frequency when rare. These protected polymorphisms continue to evolve and evolutionary branching can also occur starting from within populations of two alleles (Geritz et al. 1999). However, the study of evolution in protected polymorphisms is beyond the scope of this paper.

Evaluating second-order derivatives of invasion fitness (Eqn. 9), one can find that the cESS body size $\phi^{P}=1$ and the alleles $x_{1}^{P}, x_{2}^{P}$ that produce it, are evolutionarily attract-

ing and not invasible when $v<\frac{s}{1-s}$. Otherwise $x_{1}^{P}$ and $x_{2}^{P}$ are evolutionary branching points. Parameter $v$ determines the local curvature of competition function $\alpha_{i j, k l}$ evaluated at $\phi\left(x_{i}, x_{j}\right)=\phi\left(x_{k}, x_{l}\right)$. It determines, starting from a zero difference, how fast competition intensity starts levelling off when the difference between phenotypes increases. The parameter $s$ is not only the maximum value of function $\delta_{i j, i j}$, but also determines the local curvature of this function. This last property is more important for evolutionary attractivity and invasibility as it describes how fast the competition intensity between phenotypically identical resident individuals increases with a deviation from the cESS strategy. Using expression $\delta_{m r, r r}=1-\alpha_{m r, r r}\left(1-\delta_{r r, r r}\right)$, and writing out a Taylor expansion for this function with small mutant deviations around the cESS phenotype $\phi^{P}$, one can see that evolution will halt at a body size with value one if a mutant phenotype deviating from that cESS strategy will feel competition more severely than an individual of the cESS resident phenotype, i.e., when $\delta_{m r, r}$ decreases compared to $\delta_{r, r r}$. Evolutionary branching will occur when, for types slightly different from the cESS, competition intensity ratios level off much faster and mutants feel competition less than the residents. The P-level candidate ESS's in Fig. 2 are CSS's, evolutionarily attracting ESS's.

From second-order derivatives of invasion fitness, one finds that an I-level cESS $x^{I}$ never is an evolutionary branching point. It is either invasible and not evolutionarily attracting, such as in Fig. 2, or evolutionarily attracting and not invasible.

\section{Qualitative Types of Evolutionary Dynamics and Dependence on Model Parameters}

Pairwise Invasibility Plots are also used to study the dependence of the pattern of invasion fitnesses on ecological parameters (e.g., Geritz et al. 1999) such as parameters $v$ 


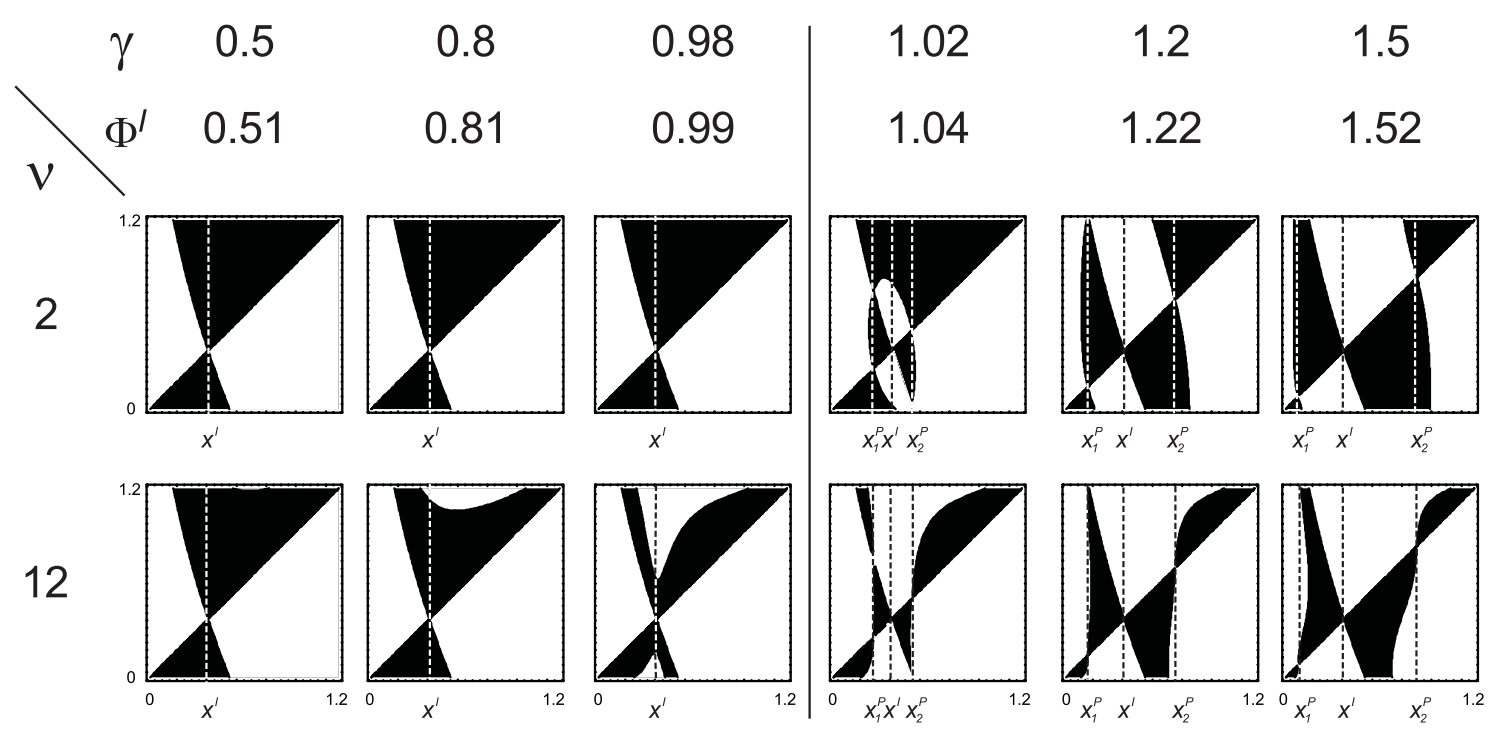

Figure 3 Diploid PIP's are shown for different combinations of competion parameter $v$ and phenotype recipe parameter $\gamma$. This parameter $\gamma$ primarily affects the maximum possible body size $\phi^{I}$. When parameter $\gamma$, which maps metabolic profit to body size, is changed, the pattern of invasion fitness changes dramatically. As long as the maximum body size $\phi^{I}$ is larger than the phenotype $\phi^{P}=1$ favoured by the population ecology, the P-level candidate ESS's $x_{1}^{P}$ and $x_{2}^{P}$ are evolutionarily attracting, otherwise the Ilevel cESS $x^{I}$ attracts. The competition parameter $v$ determines whether attracting P-level candidate ESS's are invasible or not (thus whether they are CSSs or evolutionary branching points). (phenotype and population parameters $\left.c_{1}=6, c_{2}=4, c_{3}=0.4, c_{4}=0, b_{\max }=12, s=0.9\right)$. Attracting I-level cESSs are never invasible.

and $s$ in the demographic functions (Eqns. 8 and 9). In a model with explicit genetics, diploid PIP's can also illustrate the effects of changing parameters in the phenotype recipe or of changing the phenotype recipe altogether. With changes in model parameters, existence, invasibility and attractivity of cESS's can change drastically. Figure 3 shows the effects of changing the ecological parameter $v$ and of changing the growth rate parameter $\gamma$ that appears in the phenotype recipe, on the pattern of invasion fitnesses in diploid PIP's. Changes in these parameters are sufficient to illustrate all qualitative patterns of evolutionary dynamics that occur in this model.

In the PIP's of Fig. 3, vertical dotted lines are drawn through all candidate ESS's. First of all, the growth rate parameter $\gamma$ can be that small that body size $\phi^{P}=1$ cannot be realized by the phenotype recipe (when $\gamma<1$ in Fig. 3). In that case the I-level cESS $x^{I}$ is evolutionarily attracting and not invasible. The population is stuck in this maximum body size. It cannot generate the genetic variation that would make evolution proceed towards the P-level ESS, but selection keeps it as close to it as possible. When the maximum body size for a phenotype recipe is exactly one, P-level and I-level cESS coincide at the same activity parameter value. Invasibility and attractivity are then as for the P-level cESS's discussed below (result or PIP not shown). As soon as $\gamma$ is sufficiently large in order to bring the P-level cESS within reach of the phenotype recipe, the 
I-level cESS loses attractivity and becomes invasible. Now two P-level cESS's $x^{P}{ }_{1}$ and $x_{2}^{P}$ appear that produce a body size with value one, and these are evolutionarily attracting. Invasibility of these cESS's depends on the value of competition parameter $v$. If $v$ is increased starting from a small value, P-level cESS's will change from CSS's into evolutionary branching points. For the parameter values used to produce Fig. 3, this occurs when $v>9$. The top row of Fig. 3 has a small value of $v$ that results in a pair of $\operatorname{CSS} x^{P}{ }_{1}$ and $x_{2}^{P}(v=2)$, the bottom row has a large value for this parameter $(v=12)$. The former CSS allele parameter values $x_{1}^{P}$ and $x^{P}{ }_{2}$ are now invasible by alleles with both smaller and larger total activities.

Summarizing, there are three qualitative types of evolutionary dynamics possible: $(i)$ The phenotype recipe cannot produce the body size favoured by the population ecology. Evolution proceeds towards the body size nearest to it, which corresponds to the allele activity producing maximum body size in the homozygous state. (ii) The phenotype recipe can realize the P-level cESS body size. This body size is a CSS strategy. Evolution proceeds towards one of two possible values of the activity parameter that produce this body size. (iii) The phenotype recipe can realize the P-level cESS body size. This body size is an evolutionary branching point. Evolution proceeds towards a population that is a protected polymorphism of body sizes.

\section{Overdominance in Single-Locus Allele Polymorphisms}

A population needs to contain a polymorphism of alleles at the focal metabolic profit locus or heterozygotes, overdominant or not, will not be present permanently. This section discusses when to expect overdominance in a genetic polymorphism. Two main factors contribute to the presence of genetic polymorphism in a population system. Firstly, in finite populations, a mutation-selection-drift balance establishes when selection differentials are small. Polymorphisms caused by this mechanism are in general of very similar alleles. Secondly, protected polymorphisms can occur, where the polymorphism is maintained by selection. Protected polymorphisms contain substantially different alleles. In finite populations, a mutation-selection-drift balance builds up in a protected polymorphism as well, such that it often consists of a number of separate clusters of similar alleles.

\section{Mutation-Selection-Drift Balance}

With realistic mutation rates and in finite population systems, evolutionarily stationary situations always are in a mutation-selection-drift (MSD) balance with plenty of genetic variation around the alleles predicted by adaptive dynamics. The value of invasion fitnesses for mutants appearing in a resident system become very close to zero when an 
evolving population approaches the evolutionary attractor state. Therefore, at the evolutionary attractor of a finite population system, a mutation-selection-drift balance occurs of various very similar alleles that cluster around the trait value(s) predicted by adaptive dynamics. The small selective differences between these alleles make it that selection is not effective at removing them from the population.

When the adaptive dynamics predicts an evolutionary attractor of one allele, this corresponds to a finite population with a group of alleles clustered around the value predicted by the adaptive dynamics approximation. Overdominant heterozygotes can be observed when an I-level ESS is expected. Total enzyme activity per individual then nearly produces maximum body size. In the cluster of total enzyme activities occurring, homozygous genotypes with total enzyme activities on both sides of the activity producing maximum body size will be present (Fig. 1). Their heterozygotes are phenotypically overdominant. Only at an I-level ESS or evolutionary attractor, this pattern can occur in a stable manner. We can discard I-level cESS's that are invasible and not evolutionarily attracting as a possibility for observing overdominance because such population states are not reachable from other population states. If they occur, the evolutionary system certainly evolves to a population state where overdominance cannot be observed.

At a P-level CSS, all heterozygotes have intermediate phenotypes.

\section{Polymorphic Populations}

When evolutionary branching has taken place, a polymorphic population with two resident alleles and a phenotypically overdominant heterozygote will not occur in this model.

These two allelic traits necessarily diverge after evolutionary branching. After evolutionary branching from a P-level cESS $x_{1}^{P}$ or $x_{2}^{P}$, one allele activity parameter will become smaller than the cESS value from where branching occurred, the other one larger, but both of them will be, at least initially, either larger or smaller than $x^{I}$. The body size of heterozygotes is then always intermediate between the homozygous body sizes: all total activities in individuals will remain situated on the same side of the total activity giving maximum body size.

For overdominance to evolve, one allelic trait has to change in such a way that an allele parameter crosses the I-level allelic parameter $x^{I}$. During the evolutionary process, one homozygous phenotype then first becomes equal to the maximum body size, and subsequently smaller again.

From the population dynamical recurrence equation, one can find that all possible resident populations of two alleles are protected polymorphisms. When evolutionary branching takes place from a P-level cESS, then the I-level cESS is not attracting. No allele conferring a body size that is still larger can then invade a population of homozy- 
gotes with nearly maximal body size. Such an extreme phenotype would correspond to the phenotype of the heterozygote and the nearly maximum body size to one of the homozygotes in a polymorphism that is close to straddling the I-level cESS. Since the heterozygote with maximum body size considered cannot invade the homozygous phenotype, alleles near $x^{I}$ cannot exist as a protected polymorphism. This sets up a barrier for evolving polymorphisms around the I-level cESS. Therefore, phenotypic overdominance cannot evolve from intermediate dominance by means of small evolutionary steps.

Evaluating all results in this section, one can conclude that the only possibility for observing direct phenotypic overdominance is at an individual-level evolutionary attractor. In the mutation-selection-drift balance that such a situation entails in reality, overdominant heterozygotes will occur.

\section{An Intrinsic Difference for Heterozygotes}

It is also possible to model an intrinsic trait difference or 'advantage' for heterozygous individuals. There is no evidence that such intrinsic differences are common (Lynch and Walsh 1998), but we can build a model that incorporates it and find out whether the probability of observing overdominance would change a lot in the new evolutionary dynamics.

We can for instance assume that body size not only depends on the total activity of two alleles, but also on the difference between the two allelic activities. Such an assumption results in a modified phenotype recipe $\phi$ :

$$
\phi^{\prime}\left(x_{1}, x_{2}\right)=\gamma\left[\frac{c_{1}\left(x_{1}+x_{2}\right)}{1+c_{2}\left(x_{1}+x_{2}\right)}-c_{3}\left\{1-c_{5} \frac{\left(x_{1}-x_{2}\right)^{2}}{1+\left(x_{1}-x_{2}\right)^{2}}\right\}\left(x_{1}+x_{2}\right)^{2}-c_{4}\right]
$$

Phenotype recipe $\phi^{\prime}$ (Eqn. 13) assumes that alleles have a metabolic benefit with additive effects at the level of activity, while the metabolic cost additionally depends on the difference between allele activities. The phenotype recipe of Equations (1)-(3) can be obtained from Equation (13) by setting parameter $c_{5}$ at zero. Figure 4 illustrates the effect of modifying the phenotype recipe by repeating PIP's shown in Fig. 3. Incorporating this type of intrinsic heterozygote difference into a phenotype recipe often gives the same candidate ESS's and local pattern of invasibility and attractivity as found without it (compare Figs. 3 and 4). The invasion fitness pattern does change considerably at a distance from the main diagonal, when mutant and resident alleles are very different. The existence of P-level cESS's, and the pattern of invasibility and attractivity around them always shows the same dependence on the ecological parameter $v$ and the 


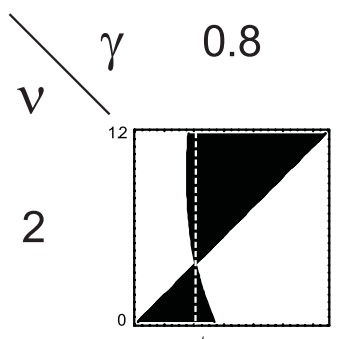

12

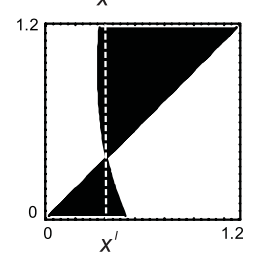

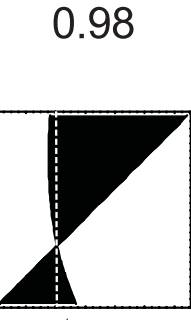

$x^{\prime}$

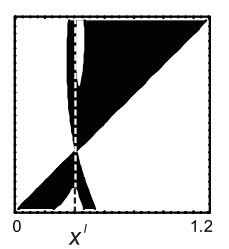

1.02

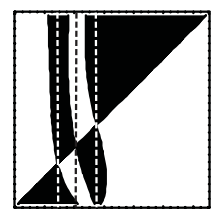

$x_{1}^{P} x^{\prime} x_{2}^{P}$

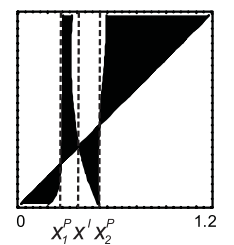

1.2
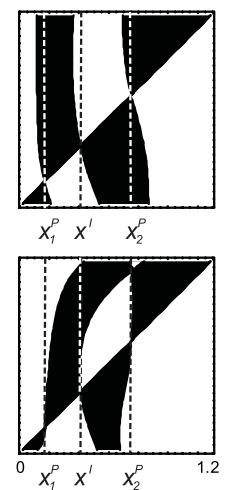

Figure 4 This figure repeats part of figure three, for a modified phenotype recipe $\phi$ ' with an intrinsic increase in body size for heterozygotes. The intrinsic difference parameter $c_{5}$ is set at $1-10^{-8}$. The pattern of candidate ESS's remains unchanged in comparison with Fig. 3: their location, local invasibility and attractivity show the same dependence on the competition parameter $v$ and the scale parameter $\gamma$. At a distance from the main diagonal, the pattern of invasion fitnesses is notably affected by the change in phenotype recipe.

growth rate parameter $\gamma$, whatever the value of the new additional parameter $c_{5}$. However, when the intrinsic difference parameter $c_{5}$ becomes very large while $\gamma$ is small, evolutionary branching can occur from an I-level cESS also (not shown in Fig. 4).

With this modified phenotype recipe $\phi^{\prime}$ (Eqn. 13), phenotypic overdominance can occur for pairs of alleles that are both either smaller or larger than $x^{I}$. The alleles do need to be substantially different before the intrinsic difference is capable of producing heterozygotes that have larger body sizes than both homoygotes. With only small differences between alleles, heterozygotes will most likely stay nearly additive. The intrinsic advantage has to be very large before one will be able to observe overdominant heterozygotes in the mutation-selection-drift balance around a P-level ESS.

\section{Evolution at Many Loci}

So far, properties of evolutionary random walks with one evolving locus are discussed. Other loci determine genetic background parameters in phenotype recipes (Eqns. 1-3 and Eqn. 13). One could also write out a phenotype recipe with explicit allele parameters for another locus. In a population with one allele at the metabolic profit locus, such as an ESS (CSS) population in the adaptive dynamics approximation, the effect of the formerly focal metabolic profit locus can be subsumed in the new genetic background parameters. It is unlikely that, when writing out phenotype recipes for all loci possibly contributing to phenotype, maxima will occur each time at intermediate values of allele parameters. Many loci must occur having partially dominant inheritance always. Equation (12) shows that evolutionary models for these loci will not have I-level ESS's, only 
P-level ESS's. Aplying that consideration to this evolutionary model, populations at an I-level ESS will often be perturbed by evolutionary events at other loci with partially dominant inheritance that do not "perceive" the I-level ESS of another locus as such. These perturbations cause changes in the genetic background parameters $c_{i}$ of Eqn. (1). From Eqn. (1), one can see that an allele substitution at another locus will change the location of the maximum in the phenotype recipe function and therefore the location of the I-level $x^{I}$. At the same time, an allele substitution an another locus displaces the population from the phenotype that is the new maximum from the viewpoint of the metabolic profit locus. Consequentially, overdominance produced by one locus will often be destroyed by an evolutionary event in another locus.

It is unlikely that such perturbations will stop as long as the population phenotype is not equal to the P-level candidate ESS phenotype, and evolution in the genetic background will bring the population phenotype each time closer to the P-level cESS phenotype. When the population eventually reaches a P-level cESS that is a CSS, evolution at the phenotypic level stops. Selective pressures on alleles determining genetic background parameters disappear: $D_{\phi_{M}} \sigma$ equals zero and every locus in the genetic background now contributes to the production of the phenotype favoured by the population ecology. As such the directional selection pressure from the population ecology disappears.

Phenotypic overdominance between some allele pairs in a mutation-selection-drift balance is expected only when the evolutionary process brings the population onto an Ilevel singular point for a certain locus, before a mutant in another locus with partially dominant effects invades and perturbs the overdominance pattern, or brings the P-level cESS within reach of the phenotype recipe. Overdominance will only be observed with appreciable likelihood when mutation rates at metabolic profit loci are much higher than at other loci, such that the population each time evolves to a phenotypic maximum for the metabolic profit loci before the next mutation in one of the other loci blurs the pattern. An at first sight different mechanism that can keep a population at an I-level ESS would be an evolutionary constraint at the phenotypic level, i.e., a mechanism that forbids a further increase in body size. However, such a constraint can be modelled as an infinitely slow mutation rate for the loci that might destroy the pattern of overdominance.

In the context of the evolution of an intrinsic advantage for heterozygotes, its probability of occurrence and evolution can be constrained. In populations with one resident allele per locus contributing to body size, modifier alleles that change the genetic background parameter $c_{5}$ in Eqn. (18) exclusively, have an invasion fitness gradient that is equal to zero. A change in parameter $c_{5}$ modifies the phenotype of heterozygotes only. Therefore, a change in the cost-reducing effect for heterozygotes goes unnoticed at the 
phenotypic level when the resident population consists of nothing but homozygotes of the resident alleles. An intrinsic advantage for heterozygotes can evolve easily only when the modifier alleles causing it have other phenotypic effects on which selection can act, during selective sweeps or in protected polymorphisms where heterozygotes are permanently present (Bürger 1983; Van Dooren 1999).

\section{Discussion}

This study applies the adaptive dynamics methodology (Dieckmann and Law 1996; Metz et al. 1996; Dieckmann 1997; Geritz et al. 1997, 1998) to a genetic model of an evolving population. The approach is similar in spirit to the streetcar theory of evolution (Hammerstein 1996). These two methods aim to identify reachable stationary states of the evolutionary process. In this case multi-locus arguments were necessary to identify the evolutionary stops or evolutionary attractors that are most likely to occur.

\section{Genetics and Adaptive Dynamics}

It is not standard in theoretical population genetics to perturb a population dynamical model with a continuous trickle of new mutants. Often the population dynamics of a system with a small and fixed number of alleles is studied, and perturbations by introducing small numbers of mutants are not considered. In adaptive dynamics, perturbing the population dynamics with mutants is the central part of an evolutionary analysis. The goal of this method is finding population dynamical systems with 'evolutionary robustness': systems that are stationary on an evolutionary timescale. Evolutionary models with sexual and diploid genetics that explicitly refer to adaptive dynamics as a method, have studied evolution among alleles at one or two loci (Kisdi and Geritz 1999, in press; Van Dooren 1999) or the evolution of a large number of loci with alleles having diallelic 0-1 effects (Dieckmann and Doebeli 1999). All of these studies have exploited the capacity of the approach to study the evolutionary process in populations that are protected polymorphisms. In this paper, genetic evolution in protected polymorphisms is not investigated. The focus is on dominance interactions between similar alleles in a group of similar alleles that are present in an evolutionarily stationary population.

Multi-locus arguments were necessary in this study to point out the existence of evolutionary attractors in a single-locus model that are likely to disappear when letting multi-locus evolution go its course. It is probably wise to introduce comparable checks in future studies of genetic evolutionary models, on the genetic robustness of evolutionarily stationary states and when estimating the likelihood of specific evolutionary trajectories. These checks can investigate the stationarity of genetic architecture in evolving populations, applying adaptive dynamics or a related methodology. Single-locus 
models surely need to be evaluated for evolutionary 'multi-locus robustness' when it is known that the phenotypic traits modelled are not always under control of a single locus. Another appropriate check is on the effects of the distribution of mutational effects. Diallelic loci are convenient for simulation, but it is unclear whether phenotypes are ever composed of a large number of freely recombining 0-1 contributions. Fact is that by restricting mutational effects to $0-1$ per allele and with free recombination, protected polymorphisms can be maintained in a large number of loci (Dieckmann and Doebeli 1999). The pattern of phenotypic variation in such a population is not clustered, but smeared out over a large range of phenotypes. This pattern is not observed in multilocus simulations where mutational effects are small and from a continuous distribution (Kisdi and Geritz 1999; Van Dooren manuscript). Models where loci have 0-1 contributions can be checked on evolutionary 'trait specification robustness' by allowing recombination to evolve, or by adding loci with mutational effects from a continuous distribution. Actually, the two proposed checks are inherent to models where the evolution of gene duplication and recombination are allowed. Building such models from simple components that are also in accordance with what is known about the genetic mechanisms involved in these phenomena, presents a major challenge.

\section{Mutation-Selection-Drift Balance}

An important message from evolutionary dynamics is that populations will spend most of their evolutionary time in situations where the selective differences between similar phenotypes are small or even negligible. In both these situations, a mutation-selectiondrift balance will establish in any real and finite system. The stationary states of the evolutionary process have such small selective differences. Selection differentials are also small at evolutionary branching points (Metz et al. 1996). This implies slow evolution and often a long waiting time for evolutionary branching (Dieckmann and Doebeli 1999). When evolutionary branching occurs, selective differences between similar types increase again afterwards. This divergent selection is a temporary phenomenon: as soon as the next evolutionary branching point or an evolutionary attractor is approached, the selective differences between similar alleles decrease again. The genetic variation that was originally present in the MSD balance at the evolutionary branching point, is in the course of this process replaced by genetic variation between the very different alleles that evolve in protected polymorphisms. Also, when the ecological setting changes quickly, such that evolutionary stationarity is lost, one can expect that selective differences between similar types will become larger temporarily. This does not need to imply that most of the genetic variation will be lost. Bürger and Lynch (1995) found that the genetic variance of a population responding to a changing environment can even increase temporarily as the result of a combination of directional and stabilising selection. However, large fluctuations of trait variances are the rule in simulations of popula- 
tions at MSD balance (Bürger and Lande 1994; Bürger and Lynch 1995; Van Dooren manuscript). A sudden decrease in genetic variation therefore does not imply that the ecology has changed.

\section{On The Likelihood of Direct Phenotypic Overdominance}

In this model for overdominance evolution, quasi-stationary states of the evolutionary process can be found when the supply of mutational variation is high for metabolic profit loci and low for other loci with modifying effects. The population can then stay a substantial amount of time near an I-level ESS (CSS) strategy, where the phenotype is maximal for the effects of a metabolic profit locus that evolves relatively fast. Only at such an individual-level ESS, overdominance can be observed. This study suggests that, in populations that are not protected polymorphisms, direct phenotypic overdominance will be most likely present $(i)$ in phenotypic traits predominantly determined by metabolic profit loci, as this should increase the chance that one of them is temporarily at an I-level ESS (CSS), (ii) when the population ecology favours an ESS that cannot be produced by the phenotype recipe. The evolving system is then developmentally constrained in a way.

In a protected polymorphism of two different clusters of alleles and an approximately homogeneous genetic background, phenotypic overdominance of heterozygotes is unlikely when there is only one phenotypic cESS that is an evolutionary branching point.

According to Crow (1952), it is doubtful that a system with overdominance at the phenotypic level from additivity at the level of catalytic activity would persist over long evolutionary periods. In this paper, Crow's arguments correspond to one specific evolutionary scenario in a more elaborate picture for the evolution of overdominance. He constructed his argument around the idea that allele dimorphisms with the heterozygote overdominant for a character that correlates with fitness, are in most cases invadable by an allele with intermediate genetic effects and a homozygous phenotype which does the same as the original heterozygote. This study confirms Crow's opinion in that respect. His argument corresponds to an evolutionary random walk that has an I-level ESS as the evolutionary attractor. In this paper, it is concluded that precisely in this case and because of the mutation-selection-drift balance that builds up, overdominance can be observed. Overdominance can appear, but probably only temporarily because substitutions at other loci destroy it very easily. Eventually, one expects to find the population-level evolutionary attractor in a population even when other modifying loci evolve relatively slowly in comparison with metabolic profit loci.

Many previously suggested mechanisms for phenotypic overdominance correspond to the mechanism modelled in this study, namely that the activity of the heterozygote is closer to the dose maximising a phenotypic trait (Hull 1952). There is no evidence for a widespread occurrence of overdominance (Mitton 1993), and the amount of studies that 
test whether these mechanisms operate as assumed has been scarce. Therefore, the nonlinear function that is mapping allelic parameters to body size in this model, remains to some extent an arbitrary choice. However, with respect to the evolution of overdominant heterozygotes, the only relevant property of such a function is that there should be a maximum phenotypic value for intermediate total activities. Many plausible nonlinear functions have this property, and the same types of evolutionary behaviour can be expected each time (Van Dooren 2000). A halfway mechanistic explanation for overdominance not investigated is physiological mosaic dominance. Mosaic dominance occurs when one allele does something the other fails to do, such that the heterozygote becomes the only one that can perform all functions. It will not persist for long either on an evolutionary timescale. One gene duplication is sufficient to put the two alleles forever together (Crow 1952).

If an intrinsic phenotypic advantage for heterozygotes would occur for some reason, it will make differences between homozygotes and heterozygotes more apparent when evolution is attracted towards an individual-level ESS. There is little supporting evidence for the presence of an unconditional advantage to heterozygotes (Lynch and Walsh 1998). In addition, I argued that they might not evolve that easily. Moreover, intrinsic differences are likely to disappear once substitutions at other loci have introduced the population level ESS-phenotype. The possibility always remains that mixing of different populations that have previously evolved independently can produce phenotypic overdominance. This is luxuriance in the words of Dobzhanky (1952), as this mixing occurs out of the ecological context of evolution.

All in all, phenotypic overdominance is expected to be found only rarely in outbreeding populations. In populations with ongoing inbreeding, there is no reason to expect that the population would stay at an individual-level candidate ESS either. Effects on viability from the expression of rare deleterious alleles will increase with inbreeding, but that should not hamper the course of evolution of the common alleles in the population. One can conclude that our a priori belief in the occurrence of direct overdominance versus associative or global overdominance should be very skewed towards associative and global overdominance.

\section{References}

Athreya, K. B., and S. Karlin 1971. On branching processes with random environments: I. extinction probabilities. Ann. Math. Stat. 42:1499-1520.

Barton, N., and M. Turelli. 1989. Evolutionary quantitative genetics: how little do we know? Ann. Rev. Ecol. Genet. 23:337-370. 
Bierne, N., S. Launey, Y. Naciri-Graven, and F. Bonhomme. 1998. Early effect of inbreeding as revealed by microsattelite analyses on Ostrea edulis larvae. Genetics 148:1893-1906.

Brown, G. C. 1991. Total cell protein concentration as an evolutionary constraint on the metabolic control distribution in cells. J. theor. Biol. 153: 195-203.

Bürger, R. (1983) Nonlinear analysis of some models for the evolution of dominance. $J$. Math. Biol. 16:269-280.

Bürger, R., and R. Lande. 1994. On the distribution of the mean and variance of a quantitative trait under mutation-selection-drift balance. Genetics 138:901-912.

Bürger, R., and M. Lynch. 1995. Evolution and extinction in a changing environment: a quantitative genetic analysis. Evolution 49: 151-163.

Christiansen, F. B. 1991. On conditions for evolutionary stability for a continuously varying character. Am. Nat. 138: 37-50.

Christiansen, F. B., and V. Loeschke. 1980. Evolution and intraspecific exploitative competition I. One-locus theory for small additive gene effects. Theor. Pop. Biol. 18:297-313.

Collet, P., and J. P. Eckmann. 1980. Iterated maps on the interval as dynamical systems. Birkhaüser, Boston.

Coulson, T. N., J. M. Pemberton, S. D. Albon, M. Beaumont, T. C. Marshall, J. Slate, F. E. Guinness, and T. H. Clutton-Brock. 1998. Microsattelites reveal heterosis in red deer. Proc.R.Soc.Lond. B 265:498-495.

Cressman, R., and W. G. S. Hines. 1984. Evolutionarily Stable Strategies of diploid populations with semi-dominant inheritance patterns. J. theor. Biol. 101:19-38.

Crow, J. F. 1952. Dominance and overdominance.. in Gowen (1952), pp. 282-297.

David, P., B. Delay, P. Berthou, and P. Jarne. 1995. Alternative models for allozymeassociated heterosis in the marine bivalve Spisula ovalis. Genetics 139:17191726.

Dieckmann, U. 1997. Can Adaptive Dynamics Invade? TREE 12:128-131.

Dieckmann, U. and M. Doebeli. 1999. On the origin of species by sympatric speciation. Nature 400: 354-357.

Dieckmann, U., and R. Law. 1996. The dynamical theory of coevolution: A derivation from stochastic ecological processes. J. Math. Biol. 34:579-612.

Diekmann, O. 1997. The many facets of evolutionary dynamics. J. Biol. Syst. 5:325329. 
Dobzhansky, Th. 1952. Nature and origin of heterosis. in Gowen (1952), pp. 218-223.

Doebeli, M. 1998. Invasion of rare mutants does not imply their evolutionary success: a counterexample from metapopulation theory. J. Evol. Biol. 11:389-401.

Eshel, I. 1983. Evolutionary and continuous stability. J. theor. Biol. 103:99-111.

Eshel, I. 1996. On the changing concept of evolutionary population stability as a reflection of a changing point of view in the quantitative theory of evolution. J. Math. Biol. 34:485-510.

Ewens, W. J. 1969. Population Genetics. Methuen \& Co Ltd, London.

Ferrière, R., and B. Cazelles. 1998. Universal power laws govern intermittent rarity in communities of interacting species. Ecology 80:1505-1521.

Ferriere, R., and M. Gatto. 1995. Lyapunov exponents and the mathematics of invasion in oscillatory and chaotic populations. Theor. Pop. Biol. 48:126-171.

Fisher, R. A. 1930. The Genetical Theory of Natural Selection. Clarendon Press, Oxford.

Geritz, S. A. H., E. Kisdi, G. Meszena, and J. A. J. Metz. 1998. Evolutionary singular strategies and the growth and branching of the evolutionary tree. Evol. Ecol. 12:35-57.

Geritz, S. A. H., E. van der Meijden, and J. A. J. Metz. 1999. Evolutionary dynamics of seed size and seedling competitive ability. Theor. Pop. Biol. 55: 324-343.

Geritz, S. A. H., J. A. J. Metz, É. Kisdi, and G. Meszéna. 1997. Dynamics of adaptation and evolutionary branching. Phys. Rev. Lett. 78, 2024-2027.

Gowen, J.W. 1952. Heterosis. Iowa State College Press, Ames Iowa.

Haccou, P., and Y. Iwasa. 1996. Invasion probability in fluctuating environments: a branching process model. Theor. Pop. Biol. 50: 254-280.

Hall, J. G., and C. Wills. 1987. Conditional overdominance at an alcohol dehydrogenase locus in yeast. Genetics 117:421-427.

Hammerstein, P. 1996. Darwinian adaptation, population genetics and the streetcar theory of evolution. J. Math. Biol. 34:511-532.

Hawkins, A. J. S., B. L. Bayne, and A. J. Day. 1986. Protein turnover, physiological energetics and heterozygosity in the blue mussel Mytilus edulis: the basis of variable age-specific growth. Proc. R. Soc. Lond. B 229:161-176.

Hofbauer, J., V. Hutson, and W. Jansen. 1987. Coexistence for systems governed by difference equations of Lotka-Volterra type. J. Math. Biol. 25:553-570. 
Houle, D. 1989. Allozyme-associated heterosis in Drosophila melanogaster. Genetics 123:789-801.

Hull, F. H. 1952. Recurrent selection and overdominance. in Gowen (1952), pp. 451473.

Kacser, H., and J.A. Burns. 1973. The control of flux. Symp. Soc. Exp. Biol. 27, 65-104.

Kacser, H., and J.A. Burns. 1981. The molecular basis of dominance. Genetics 97: 639666.

Kisdi, É., and S. A. H. Geritz. 1999. Adaptive Dynamics in allele space: evolution of genetic polymorphism by small mutations in a heterogeneous environment. Evolution 53: 993-1008.

Kisdi, É., and S. A. H. Geritz. in press. Evolutionary branching and sympatric speciation in diploid populations. In Metz and Dieckmann (in press).

Kisdi, É., and G. Meszéna. 1992. Density dependent life history evolution in fluctuating environments. In C. W. Clark, J. Yoshimura, eds. Adaptation in a stochastic environment. Lecture Notes in Biomathematics 98.

Koehn, R. K. 1991. The cost of enzyme synthesis in the genetics of energy balance and physiological performance. Biol. J. Linn. Soc. 44:231-247.

Lynch, M., and B. Walsh. 1998. Genetics and analysis of quantitative traits. Sinauer Associates, Sunderland MA.

Matessi, C., and C. D. Di Pasquale. 1996. Long-term evolution of multilocus traits. J. Math. Biol. 34:613-653.

Maynard Smith, J. 1981. Will a sexual population evolve to an ESS? Am. Nat. 117:10151018.

Maynard Smith, J. 1982. Evolution and the Theory of Games. Cambridge University Press.

Maynard Smith, J. 1998. Evolutionary Genetics, second edition. Oxford University Press.

Metz, J. A. J., and U. Dieckmann. in press. Elements of Adaptive Dynamics. Cambridge University Press.

Metz, J. A. J., and O. Diekmann. 1986. A gentle introduction to structured population models: three worked examples. in J. A. J. Metz and O. Diekmann, (eds.) The Dynamics of Physiologically Structured Populations. Lecture Notes in Biomathematics 68, Springer, Berlin, pp.-3-45. 
Metz, J. A. J., S. A. H. Geritz, G., Meszéna, F. J. A. Jacobs, and J. S. van Heerwaarden. 1996. Adaptive dynamics: a geometrical study of the consequences of nearly faithful reproduction. in S. J. Van Strien and S. M. Verduyn Lunel, (eds.) Stochastic and Spatial Structures of Dynamical Systems. KNAW Verhandelingen, North Holland, pp.183-231.

Metz, J. A. J., R. M. Nisbet, and S. A. H. Geritz. 1992. How should we define fitness for general ecological scenarios? TREE 7, 198-202.

Mitton, J. B. 1993. Theory and data pertinent to the relationship between heterozygosity and fitness. in N. W. Thornhill, ed. The Natural History of Inbreeding and Outbreeding. The University of Chicago Press., pp. 17-41.

Pogson, G.H., and S. E. Fevolden. 1998. DNA heterozygosity and growth rate in the Atlantic cod Gadus morhua (L). Evolution 52:915-920.

Prout, T. 1968. Sufficient conditions for multiple niche polymorphism. Am. Nat. 102: 493-496.

Rand, D. A., H. B. Wilson, and J. M. McGlade. 1994. Dynamics and evolution: evolutionary stable attractors, invasion exponents and phenotype dynamics. Phil. Trans. Roy. Soc. Lond. B 343:261-283.

Ricker, W. E. 1954. Stock and recruitment. J. Fish. Res. Bd. Can. 11:559-623.

Royama, P. 1992. Analytical Population Dynamics. Chapman \& Hall, London.

Savolainen, O., and P. Hedrick. 1995. Heterozygosity and fitness: no association in scots pine. Genetics 140:755-766.

Schull, G. H. 1914. Duplicate genes for capsule form in Bursa bursa-pastoris. Zeitschr. indukt. Abstamm.-u. Vererbungsl. 12:97-149.

Strauss, S. H. 1986. Heterosis at allozyme loci under inbreeding and crossbreeding in Pinus attenuata. Genetics 113:115-134.

Turchin, P. 1995. Population regulation: old arguments and a new synthesis. Pp. 19-40 in N. Cappuccino and P. W. Price (eds.) Population dynamics: New Approaches and Synthesis. Academic Press, pp.19-40.

Van Dooren, T. J. M. 1999. The evolutionary ecology of dominance-recessivity. $J$. theor. Biol. 198: 519-532.

Van Dooren, T. J. M. 2000. Phenotype Recipes in Evolutionary Dynamics, PhD. Thesis. University of Antwerp, Belgium.

Van Dooren, T. J. M. in press. Adaptive dynamics for Mendelian genetics. In Metz and Dieckmann (in press). 
Van Tienderen, P. H.. and G. De Jong. 1986. Sex ratio in the haystack model: Polymorphism may occur. J. theor. Biol. 122:69-81.

von Bertalanffy, L. 1934. Untersuchungen über die gesetzlichkeit des Wachstums, I Teil Allgemeine grundlagen der Theorie: mathematische und physiologische Gesetzlichkeiten der Wachstums bei Wassertieren. Wilhelm Roux' Arch. Entwicklungsmech. Org. 131: 613-652.

Xiao, J., L. Jiming, Y. Longping. and S. D. Tanksley. 1995. Dominance is the major genetic basis of heterosis in rice as revealed by QTL analysis using molecular markers. Genetics 140: 745-754.

Zouros, E., M. Romero-Dorey, and A. L. Mallet. 1988. Heterozygosity and growth in marine bivalves: further data and possible explanations. Evolution 42: 1332-1341. 


\section{Appendix A: Population Dynamics}

The derivation of the Ricker map, as in Royama (1992) and Leitner (1998), is repeated for a genetically explicit model and allowing for genetic polymorphism. The population dynamics is described in terms of alleles and not in terms of genotype numbers.

If we let the size of the population dynamical system, which can be understood as the total number of patches, become very large, the population dynamics of each allele is accurately described by a mean field recurrence equation for the average number of alleles per patch (this is a a measure of local allele population density). $X_{i, t}$ is the mean number of alleles $x_{i}$ settling per patch at time $t$. A mean field equation for $X_{i, t}$ has the form of Equation (A.1). The average number of alleles $x_{i}$ entering a patch at time $t+1$, is the average number of alleles that entered one timestep before, times a factor $B_{i, t}$ that depends on the specific allele $x_{i}$ and on the population composition at time $t$.

$$
X_{i, t+1}=B_{i, t} X_{i, t}
$$

Individuals can be of different genotypes and phenotypes. Genotypes are indexed $i j$ (or $k l$ ) with $i, j(k, l)=1, \ldots ., n$. The mean number of individuals per patch in generation $t$ and with genotype indexation $i j$ is $N_{i j, t}\left(N_{i j,} t=N_{j i, t}\right)$, such that the expected number of heterozygous individuals containing alleles $x_{i}$ and $x_{j}$ is $2 N_{i j, t}$. The average total number of gametes produced by the individuals $N_{i j, t}$ that survive passage through the gamete pool is $b_{i j, t}$, of which one half is of type $i$. Therefore, the average total number of alleles $x_{i}$ produced per patch, subsequently passing through the gamete pool and entering the next round of reproduction is

$$
X_{i, t+1}=\sum_{j=1}^{n} \frac{1}{2}\left(b_{i j, t}+b_{j i, t}\right)=\sum_{j=1}^{n} b_{i j, t}
$$

since $b_{i j, t}=b_{j i, t}$.

An individual-based scenario is used to derive expression for $b_{i j, t}$ and for $B_{i, t}$. (Equations A.3 to A.5). Because of random mating and settlement, the number of individuals with genotype index $i j$ in a patch before reproduction is Poisson distributed with parameter $N_{i j, t}=\frac{p_{i, t} p_{j, t} X_{t}}{2}$, where $p_{i, t}, p_{j, t}$ are the frequencies of alleles $x_{i}, x_{j}$ in the gamete pool and $X_{t}$ is the total number of gametes that will settle divided by the number of patches. $X_{t} / 2$ then is the expected total number of zygotes per patch. Equation (A.3) gives the expression for the average total number of offspring gametes $b_{i j, t}$ produced by 
the individuals in the patch with genotype index $i j$.

$$
b_{i j, t}=b_{\max } \sum_{q^{\prime}=1}^{\infty} q^{\prime} \frac{\delta_{i j, i j}^{q^{\prime}-1} \exp \left(-N_{i j, t}\right)\left(N_{i j, t}\right)^{q^{\prime}}}{q^{\prime} !} \prod_{\substack{k, l=1 \\ k l \neq i j}}^{n} \sum_{q=0}^{\infty} \frac{\delta_{i j, k l}{ }^{q} \exp \left(-N_{k l, t}\right)\left(N_{k l, t}\right)^{q}}{q !}
$$

All averages in Eqn. (A.3) are taken over the Poisson distributions of numbers of individuals of different types. The zero term of the Poison distribution is missing from the first summation. When a type of individual is absent, it cannot produce gametes. For each number of individuals $i j$ possibly present, the first summation includes the number of parent individuals $q$ ' and the competition effect from individuals that have the same genotype index $\delta_{i j, i j}^{q^{\prime}-1}$. The density-dependent exponent of $\delta_{i j, i j}$ is decreased by one because individuals is assumed not to affect/decrease their own reproduction. Each genotype $k l$ different from $i j$ decreases the total number of gametes by a factor $\delta_{i j, k l}{ }^{q}$. The average is taken over the Poisson distribution for each type of individual with an index different from $i j$. The expression for offspring production (Eqn. A.3) can be rearranged to give

$$
b_{i j, t}=b_{\max } \frac{p_{i, t} p_{j, t} X_{t}}{2} \prod_{k, l=1}^{n}\left[e^{-N_{k l, t}} \sum_{q=0}^{\infty} \frac{\left(\delta_{i j, k l} N_{k l, t}\right)^{q}}{q !}\right]
$$

where $k l$ are now all possible genotypes including $i j$. The rearrangement is obtained by changing the indexation of the first summation in Eqn (A.3), and by substituting total number of alleles and allele frequencies for $N_{i j, t}$. Observe that all summation series in Eqn. (A.4) are powers of the number $e$. From Eqn. (A.4) we can derive an expression for $B_{i, t}$ (Equation A.5), substituting powers of $e$ for the summations and dividing Eqn. (A.4) by $X_{i, t}=p_{i, t} X_{t}$.

$$
B_{i, t}=\frac{b_{\max }}{2} \sum_{j} p_{j, t} e^{-\left(1-\delta_{i j, i j}\right) \sum_{k=1, l=1}^{n} \alpha_{i j, k l} \frac{p_{k, t} p_{l, t} X_{t}}{2}} \text { with } \alpha_{i j, k l}=\frac{1-\delta_{i j, k l}}{1-\delta_{i j, i j}}
$$

When there is only one allele in the population, this equation becomes the Ricker reproduction curve (Ricker 1954). For a mutant allele appearing in such a system, the mutant allele frequency is nearly zero and the resident allele frequency approximately one, such that the local density of mutant alleles can be omitted from expression (A.5). 


\section{Appendix B: Invasion Fitness}

From Equations (4), (5) and (8) we can derive expressions for the invasion fitness $\zeta\left(x_{m}\right.$, $x_{r}$ ) of a mutant allele in a population with one resident allele. First of all, we can replace the ratio of mutant population densities in Eqn. (8) by a product of factors $B_{m, \tau}$ (Eqn. B.1).

$$
\zeta\left(x_{m}, x_{r}\right)=\lim _{t \rightarrow \infty} \frac{1}{t} \ln \prod_{\tau=0}^{t-1} B_{m, \tau}
$$

Substituting the mean field expression for this reproduction rate (Eqn. 5) into equation (B.1) and a bit of rearrangement gives invasion fitness expression (B.2).

Invasion fitness has to be zero when the mutant is equal to the resident allele type, i.e., when we substitute indices $r$ for indices $m$. Using that, we can solve for the longterm average of resident population densities appearing at the right end of expression (B.2), yielding Equation (B.3).

$$
\lim _{t \rightarrow \infty} \frac{1}{t} \sum_{\tau=0}^{t-1} X_{r, \tau}=\frac{2}{1-\delta_{r r, r r}} \ln \frac{b_{\max }}{2}
$$

Substituting Eqn. (B.3) into Eqn. (B.2) allows us to write invasion fitness $\zeta\left(x_{m}, x_{r}\right)$ in terms of the demographic functions that depend on the phenotypes of mutant and resident individuals. This gives the invasion fitness expression of Eqn. (9) (the derivation went as in Metz et al. 1992). Note that the sign of invasion fitness is not affected by the qualitative type of the resident population dynamics. A change in the fecundity parameter $b_{\max }$ that determines the qualitative regime of the population dynamics (stable equilibrium or non-equilibrium dynamics), will change the absolute value of invasion fitness, but not its sign.

Because populations of mutants originate from a single mutant allele, their initial dynamics must suffer from demographic stochasticity and is better described as a branching process approximation. Even then, irrespective of whether the resident dynamics has a stable population dynamical equilibrium or not, invasion fitness calculated from the mean field process is used to estimate the probability that the mutant population goes extinct (Ewens 1969; Athreya and Karlin 1971; Haccou and Iwasa 1996). Only when invasion fitness is larger than zero, the probability of extinction is smaller than one. 